\title{
Dimensionality and the stability of the Brunn-Minkowski inequality
}

\author{
RONEN ELDAN AND Bo`AZ KLARTAG
}

\begin{abstract}
We prove stability estimates for the Brunn-Minkowski inequality for convex sets. As opposed to previous stability results, our estimates improve as the dimension grows. In particular, we obtain a non-trivial conclusion for high dimensions already when

$$
\operatorname{Vol}_{n}\left(\frac{K+T}{2}\right) \leq 5 \sqrt{\operatorname{Vol}_{n}(K) \operatorname{Vol}_{n}(T)} .
$$

Our results are equivalent to a thin shell bound, which is one of the central ingredients in the proof of the central limit theorem for convex sets.
\end{abstract}

\section{Introduction}

The Brunn-Minkowski inequality states, in one of its normalizations, that

$$
\operatorname{Vol}_{n}\left(\frac{K+T}{2}\right) \geq \sqrt{\operatorname{Vol}_{n}(K) \operatorname{Vol}_{n}(T)}
$$

for any compact sets $K, T \subset \mathbb{R}^{n}$, where $(K+T) / 2=\{(x+y) / 2: x \in K, y \in T\}$ is half of the Minkowski sum of $K$ and $T$, and where $\mathrm{Vol}_{n}$ stands for the Lebesgue measure in $\mathbb{R}^{n}$. Equality in (1.1) holds if and only if $K$ is a translate of $T$ and both are convex, up to a set of measure zero.

The literature contains various stability estimates for the Brunn-Minkowski inequality, which imply that when there is almost-equality in (1.1), then $K$ and $T$ are almost-translates of each other. Such estimates appear in Diskant [8], in Groemer [13], and in Figalli, Maggi and Pratelli [11,12]. We recommend Osserman [20] for a general survey on the stability of geometric inequalities.

All of the stability results that we found in the literature share a common feature: Their estimates deteriorate quickly as the dimension increases. For instance, suppose that $K, T \subset \mathbb{R}^{n}$ are convex sets with

$$
\operatorname{Vol}_{n}(K)=\operatorname{Vol}_{n}(T)=1 \quad \text { and } \quad \operatorname{Vol}_{n}\left(\frac{K+T}{2}\right) \leq 5 .
$$

Supported in part by the Israel Science Foundation and by a Marie Curie Reintegration Grant from the Commission of the European Communities.

Received November 11, 2011; accepted in revised form September 5, 2012. 
The present stability estimates do not seem to imply much about the proximity of $K$ to a translate of $T$ under the assumption (1.2). Only if the constant " 5 " in (1.2) is replaced by something like $1+1 / n$ or so, then the results of Figalli, Maggi and Pratelli [12] can yield meaningful information. The goal of this note is to raise the possibility that the stability of the Brunn-Minkowski inequality actually improves as the dimension increases. In particular, we would like to deduce from (1.2) that

$$
\left|\frac{\int_{K} p\left(x-b_{K}\right) d x}{\int_{T} p\left(x-b_{T}\right) d x}-1\right| \ll 1
$$

for a family of non-negative functions $p$, when the dimension $n$ is high. Here, $b_{K}$ and $b_{T}$ denote the barycenters of $K$ and $T$ respectively. Furthermore, in some nontrivial cases we may conclude (1.3) even when the constant " 5 " in (1.2) is replaced by an expression that grows with the dimension, such as $\log n$ or $n^{\alpha}$ for a small universal constant $\alpha>0$.

In this note we take the first steps towards a dimension-sensitive stability theory for the Brunn-Minkowski inequality. First, let us focus on the simplest case in which $p(x)$ in (1.3) is a quadratic polynomial. In fact, we are interested mainly in expressions related to the quadratic form

$$
q_{K}(x)=\frac{1}{\operatorname{Vol}_{n}(K)} \int_{K}\langle x, y\rangle^{2} d y-\left(\frac{1}{\operatorname{Vol}_{n}(K)} \int_{K}\langle x, y\rangle d y\right)^{2} \quad\left(x \in \mathbb{R}^{n}\right)
$$

where $\langle\cdot, \cdot\rangle$ is the standard scalar product in $\mathbb{R}^{n}$. The inertia form of the bounded, open set $K \subset \mathbb{R}^{n}$ is defined as

$$
p_{K}(x)=\sup \left\{\langle x, y\rangle^{2}: q_{K}(y) \leq 1\right\} .
$$

Note that $p_{K}$ is a positive-definite quadratic form in $\mathbb{R}^{n}$. We say that $K \subset \mathbb{R}^{n}$ is isotropic when the barycenter of $K$ lies at the origin and $q_{K}(x)=|x|^{2}=\langle x, x\rangle$ for all $x$. In this case, also $p_{K}(x)=|x|^{2}$. It is easy to see that any bounded, open set $K \subset \mathbb{R}^{n}$ has an affine image which is isotropic.

A convex body in $\mathbb{R}^{n}$ is a bounded, open convex set. For a convex body $K \subset$ $\mathbb{R}^{n}$ we denote by $\mu_{K}$ the uniform probability measure on $K$. Our first stability result is as follows:

Theorem 1.1. Let $K, T \subset \mathbb{R}^{n}$ be convex bodies and let $R \geq 1$. Assume that

$$
\operatorname{Vol}_{n}\left(\frac{K+T}{2}\right) \leq R \sqrt{\operatorname{Vol}_{n}(K) \operatorname{Vol}_{n}(T)}
$$

Let $p(x)=p_{K}(x)$ be the inertia form of $K$ defined in (1.4) and (1.5). Then,

$$
\left|\frac{\int_{T} p\left(x-b_{T}\right) d \mu_{T}(x)}{\int_{K} p\left(x-b_{K}\right) d \mu_{K}(x)}-1\right| \leq C \frac{R^{\alpha_{2}}}{n^{\alpha_{1}}} .
$$

Here $C, \alpha_{1}, \alpha_{2}>0$ are universal constants, and $b_{K}, b_{T}$ are the barycenters of $K, T$ respectively. 
See Theorem 4.6 below for explicit bounds on the universal constants $\alpha_{1}, \alpha_{2}$ from Theorem 1.1. Our interest in the inertia form $p_{K}$ stems from the central limit theorem for convex sets, see $[9,14]$ for background reading. As we shall explain in Proposition 6.4 below, Theorem 1.1 implies the bound

$$
\sigma_{n} \leq C n^{1 / 2-\alpha_{1}}
$$

where $\sigma_{n}$ is the thin shell parameter from [10], and $C>0$ is a universal constant and $\alpha_{1}>0$ is the constant from Theorem 1.1. In fact, Theorem 4.6 and (4.25) below show that the inequality (1.7) is essentially an equivalence. Consequently, the universal constant $\alpha_{1}$ from Theorem 1.1 is intimately connected with the thin shell parameter $\sigma_{n}$. The question of whether $\sigma_{n}$ is bounded by a universal constant is currently one of the central problems in high-dimensional convex geometry.

Next, we address the task of finding a larger class of functions $p$ for which bounds such as (1.3) hold true. Suppose that $\mu_{1}$ and $\mu_{2}$ are two Borel probability measures on $\mathbb{R}^{n}$. A Borel probability measure $\gamma$ on $\mathbb{R}^{n} \times \mathbb{R}^{n}$ is called a coupling of $\mu_{1}$ and $\mu_{2}$ if $\left(P_{1}\right)_{*}(\gamma)=\mu_{1}$ and $\left(P_{2}\right)_{*}(\gamma)=\mu_{2}$ where $P_{1}(x, y)=x$ and $P_{2}(x, y)=y$. Here, $\left(P_{i}\right)_{*}(\mu)$ denotes the push-forward of $\mu$ under the map $P_{i}$ for $i=1,2$. For two Borel probability measures $\mu_{1}$ and $\mu_{2}$ on $\mathbb{R}^{n}$ and for $1 \leq p<\infty$, we set

$$
W_{p}\left(\mu_{1}, \mu_{2}\right)=\inf _{\gamma}\left(\int_{\mathbb{R}^{n} \times \mathbb{R}^{n}}|x-y|^{p} d \gamma(x, y)\right)^{1 / p}
$$

where the infimum runs over all couplings $\gamma$ of $\mu_{1}$ and $\mu_{2}$. This is precisely the $L^{p}$ Monge-Kantorovich-Wasserstein transportation distance between $\mu_{1}$ and $\mu_{2}$. See, e.g., Villani's book [22] for more information about this metric. Note that for any 1-Lipschitz function $\varphi: \mathbb{R}^{n} \rightarrow \mathbb{R}$,

$$
\left|\int_{\mathbb{R}^{n}} \varphi(x) d \mu_{1}(x)-\int_{\mathbb{R}^{n}} \varphi(x) d \mu_{2}(x)\right| \leq W_{1}\left(\mu_{1}, \mu_{2}\right) \leq W_{2}\left(\mu_{1}, \mu_{2}\right) .
$$

In fact, the assumption that $\varphi$ is 1-Lipschitz may typically be weakened. For instance, when $\varphi$ is convex or concave, it is well-known that

$$
\left|\int_{\mathbb{R}^{n}} \varphi d \mu_{1}-\int_{\mathbb{R}^{n}} \varphi d \mu_{2}\right| \leq W_{2}\left(\mu_{1}, \mu_{2}\right) \cdot \sqrt{\max \left\{\int_{\mathbb{R}^{n}}|\nabla \varphi|^{2} d \mu_{1}, \int_{\mathbb{R}^{n}}|\nabla \varphi|^{2} d \mu_{2}\right\}} .
$$

Theorem 1.2. Let $K, T \subset \mathbb{R}^{n}$ be convex bodies whose barycenters lie at the origin and let $R \geq 1$. Suppose that

$$
\operatorname{Vol}_{n}\left(\frac{K+T}{2}\right) \leq R \sqrt{\operatorname{Vol}_{n}(K) \operatorname{Vol}_{n}(T)} .
$$

Assume that $K$ is isotropic. Then,

$$
\frac{W_{2}\left(\mu_{K}, \mu_{T}\right)}{\sqrt{n}} \leq C n^{-1 / 4} \sqrt{\sigma_{n}} R^{5 / 2} \leq \tilde{C} \frac{R^{5 / 2}}{n^{\alpha}},
$$

where $\alpha, C, \tilde{C}>0$ are universal constants. 
Theorem 1.2 combined with the inequality (1.8) entails the bound (1.3) in the case where, for instance, $p(x)=\|x\|^{q}$ for various norms $\|\cdot\|$ in $\mathbb{R}^{n}, q \geq 0$ and $R \ll n^{c}$. Additionally, the estimate (1.9) implies the non-trivial bound (1.6) via (1.8). We do not know the optimal value of the exponent $\alpha$ in Theorem 1.2. We know more in the particular case of unconditional convex bodies. A convex body in $\mathbb{R}^{n}$ is said to be unconditional if

$$
\left(x_{1}, \ldots, x_{n}\right) \in K \quad \Longleftrightarrow \quad\left( \pm x_{1}, \ldots, \pm x_{n}\right) \in K
$$

for all $\left(x_{1}, \ldots, x_{n}\right) \in \mathbb{R}^{n}$ and for all possible choices of signs. In other words, $K$ is invariant under coordinate reflections. For unconditional convex bodies, Theorem 1.2 may be sharpened as follows:

Theorem 1.3. Let $K, T \subset \mathbb{R}^{n}$ be unconditional convex bodies, and let $R \geq 1$. Assume that $K$ is isotropic and that

$$
\operatorname{Vol}_{n}\left(\frac{K+T}{2}\right) \leq R \sqrt{\operatorname{Vol}_{n}(K) \operatorname{Vol}_{n}(T)}
$$

Then

$$
W_{2}\left(\mu_{K}, \mu_{T}\right) \leq C(R-1)^{5 / 2} \log n,
$$

where $C>0$ is a universal constant.

Thus, in the unconditional case, the exponent $\alpha$ from Theorem 1.2 is essentially $1 / 2$, up to logarithmic factors. When substituting $\varphi(x)=|x|^{2}$ in (1.8) and using (1.10), we conclude that for any $K, T \subset \mathbb{R}^{n}$ as in Theorem 1.3,

$$
\left.\left|\int_{K}\right| x\right|^{2} d \mu_{K}-\int_{T}|x|^{2} d \mu_{T} \mid \leq C \sqrt{n} \cdot \log n \cdot(R-1)^{5}
$$

(in order to use (1.8) we also need a crude estimate for $\int_{T}|x|^{2} d \mu_{T}(x)$, hence we applied Corollary 2.4 to obtain such an estimate). In view of (1.11) and Proposition 6.4 below, we match (up to logarithmic factors) the best bounds for the width of the thin spherical shell for unconditional convex bodies proven in [15].

The structure of the remainder of this note is as follows: In the next section we establish some well-known facts about one-dimensional log-concave measures. In Section 3 we prove Theorem 1.1 and in Section 4 we prove Theorem 1.2. Section 5 is dedicated to attaining some inequalities related to one-dimensional transportation of measure. In Section 6, using these inequalities, we prove Theorem 1.3.

Throughout this note, we write $c, C, \tilde{c}$ etc. for various positive universal constants, whose value may change from one line to the next. We usually use uppercase $C$ to denote universal constants that we consider "sufficiently large", and lower-case $c$ to denote universal constants that are "sufficiently small". We write $\log$ for the natural logarithm. By "measurable" we always mean Borel-measurable. 


\section{Background about log-concave densities on the line}

In this section we recall some facts, all of which are well-known to experts, about log-concave densities. A function $\rho: \mathbb{R}^{n} \rightarrow[0, \infty)$ is log-concave if for any $x, y \in \mathbb{R}^{n}$,

$$
\rho(\lambda x+(1-\lambda) y) \geq \rho(x)^{\lambda} \rho(y)^{1-\lambda} \quad \text { for all } 0<\lambda<1 .
$$

A probability measure or a random variable are called log-concave if they posses a $\log$-concave density. Let $\mu$ be a log-concave probability measure on $\mathbb{R}$, whose log-concave density is denoted by $\rho: \mathbb{R} \rightarrow[0, \infty)$. Write

$$
\Phi(t)=\mu((-\infty, t])=\int_{-\infty}^{t} \rho(s) d s \quad(t \in \mathbb{R}) .
$$

A nice characterization of log-concavity that we learned from Bobkov [3] is that $\mu$ is log-concave if and only if the function

$$
t \mapsto \rho\left(\Phi^{-1}(t)\right) \quad t \in[0,1]
$$

is a concave function. This characterization lies at the heart of the proof of the following Poincaré-type inequality which appears as Corollary 4.3 in Bobkov [2]:

Lemma 2.1. Let $\mu$ be a log-concave probability measure on the real line, and set

$$
\operatorname{Var}(\mu)=\int x^{2} d \mu(x)-\left(\int x d \mu(x)\right)^{2}
$$

for the variance of $\mu$. Then for any smooth function $f$ with $\int f d \mu=0$,

$$
\int_{\mathbb{R}} f^{2}(t) d \mu(t) \leq 12 \operatorname{Var}(\mu) \int_{\mathbb{R}}\left|f^{\prime}(t)\right|^{2} d \mu(t) .
$$

Further information about log-concave densities on the line is provided by the following standard lemma.

Lemma 2.2. Let $f: \mathbb{R} \rightarrow[0, \infty)$ be a log-concave probability density. Denote $b=\int x f(x) d x$, the barycenter of the density $f$, and let $\sigma^{2}$ be the variance of the random variable whose density is $f$. Then, for any $t \in \mathbb{R}$,

(a) $f(t) \leq \frac{C}{\sigma} \exp (-c|t-b| / \sigma)$; and

(b) If $|t-b| \leq c \sigma$, then $f(t) \geq \frac{c}{\sigma}$.

Here, $c, C>0$ are universal constants. 
Proof. Part (a) is the content of Lemma 3.2 in Bobkov [4]. In order to prove (b), we show that for some $t_{0} \geq b+c_{0} \sigma$,

$$
f\left(t_{0}\right) \geq 1 /\left(10 C_{1} \sigma\right)
$$

with $c_{0}=1 /(10 C), C_{1}=c^{-1} \log (10 C / c)$ where here $c, C$ are the constants from part (a). Indeed, if there is no such $t_{0}$, then from (a),

$$
\begin{aligned}
\int_{b}^{\infty} f(t) d t & \leq \int_{b}^{b+c_{0} \sigma} \frac{C}{\sigma} d t+\int_{b+c_{0} \sigma}^{b+C_{1} \sigma} \frac{d t}{10 C_{1} \sigma}+\int_{b+C_{1} \sigma}^{\infty} \frac{C}{\sigma} \exp (-c|t-b| / \sigma) d t \\
& \leq \frac{3}{10}<\frac{1}{e},
\end{aligned}
$$

in contradiction to Grünbaum's inequality (see, e.g., [4, Lemma 3.3]). By symmetry, there exists some $t_{1} \leq b-c_{0} \sigma$ with

$$
f\left(t_{1}\right) \geq 1 /\left(10 C_{1} \sigma\right) .
$$

From log-concavity, $f(t) \geq 1 /\left(10 C_{1} \sigma\right)$ for $t \in\left[t_{1}, t_{0}\right]$, and (b) is proven since $\left[t_{1}, t_{0}\right] \supseteq\left[b-c_{0} \sigma, b+c_{0} \sigma\right]$.

The following lemma is essentially a one-dimensional, functional version of Theorem 1.1. The lemma states, roughly, that if the supremum-convolution of two log-concave probability densities has a bounded integral, then their respective variances cannot be too far from each other.

Lemma 2.3. Let $X, Y$ be random variables with corresponding densities $f_{X}, f_{Y}$ and variances $\sigma_{X}^{2}, \sigma_{Y}^{2}$. Assume that $f_{X}$ and $f_{Y}$ are log-concave. Define

$$
h(t)=\sup _{s \in \mathbb{R}} \sqrt{f_{X}(t+s) f_{Y}(t-s)},
$$

a supremum-convolution of $f_{X}$ and $f_{Y}$. Then,

$$
\int_{\mathbb{R}} h(t) d t \geq c \sqrt{\max \left\{\frac{\sigma_{X}}{\sigma_{Y}}, \frac{\sigma_{Y}}{\sigma_{X}}\right\}}
$$

where $c>0$ is a universal constant.

Proof. The function $h$ is clearly measurable (it is even log-concave). It follows from Lemma 2.2(b) that there exist intervals $I_{X}, I_{Y}$ such that

$$
\text { Length }\left(I_{X}\right) \geq c \sigma_{X}, \quad \operatorname{Length}\left(I_{Y}\right) \geq c \sigma_{Y}
$$

and,

$$
f_{X}(t) \geq \frac{c}{\sigma_{X}}, \quad \forall t \in I_{X} \quad ; \quad f_{Y}(s) \geq \frac{c}{\sigma_{Y}}, \quad \forall s \in I_{Y}
$$


Combining this with (2.2), we learn that there exists an interval $I_{Z}$ with Length $\left(I_{Z}\right) \geq c\left(\sigma_{X}+\sigma_{Y}\right) / 2$ such that,

$$
h(t) \geq \frac{c}{\sqrt{\sigma_{X} \sigma_{Y}}}, \quad \forall t \in I_{Z}
$$

This implies,

$$
\int_{\mathbb{R}} h(t) d t \geq \int_{I_{Z}} h(t) d t \geq \frac{c^{2}}{2} \frac{\sigma_{X}+\sigma_{Y}}{\sqrt{\sigma_{X} \sigma_{Y}}} \geq \frac{c^{2}}{2} \sqrt{\max \left\{\frac{\sigma_{X}}{\sigma_{Y}}, \frac{\sigma_{X}}{\sigma_{Y}}\right\}}
$$

which completes the proof.

Recall the definition (1.4) of the inertia form $q_{K}(x)$ associated with a convex body $K \subset \mathbb{R}^{n}$. As a corollary of Lemma 2.3, we have

Corollary 2.4. Let $R>1$ and let $K, T \subset \mathbb{R}^{n}$ be convex bodies such that

$$
\operatorname{Vol}_{n}\left(\frac{K+T}{2}\right) \leq R \sqrt{\operatorname{Vol}_{n}(K) \operatorname{Vol}_{n}(T)}
$$

Then,

$$
\frac{1}{C R^{4}} q_{K}(x) \leq q_{T}(x) \leq C R^{4} q_{K}(x) \quad \text { for all } x \in \mathbb{R}^{n}
$$

where $C>0$ is a universal constant.

Proof. Fix a unit vector $\theta \in \mathbb{R}^{n}$. Let $\tilde{X}, \tilde{Y}$ be random vectors distributed uniformly on $K, T$ respectively, and define $X=\langle\tilde{X}, \theta\rangle$ and $Y=\langle\tilde{Y}, \theta\rangle$. Observe that

$$
q_{K}(\theta)=\operatorname{Var}(X), \quad q_{T}(\theta)=\operatorname{Var}(Y) .
$$

In order to prove (2.3), it suffices to show that

$$
\max \left\{\frac{\operatorname{Var}(X)}{\operatorname{Var}(Y)}, \frac{\operatorname{Var}(Y)}{\operatorname{Var}(X)}\right\} \leq C R^{4} .
$$

Denote the respective densities of $X, Y$ by $f_{X}, f_{Y}$. The Prékopa-Leindler theorem (see, e.g., the first pages of Pisier [21]) implies that $f_{X}$ and $f_{Y}$ are log-concave. Furthermore, using the Prékopa-Leindler theorem again we derive,

$$
\operatorname{Vol}_{n}\left(\frac{K+T}{2}\right) \geq \int_{\mathbb{R}} \sup _{s \in \mathbb{R}} \sqrt{f_{X}(t-s) \operatorname{Vol}_{n}(K) f_{Y}(t+s) \operatorname{Vol}_{n}(T)} d t .
$$

Hence,

$$
\int_{\mathbb{R}} \sup _{s \in \mathbb{R}} \sqrt{f_{X}(t-s) f_{Y}(t+s)} d t \leq R .
$$

Plugging this into Lemma 2.3 we deduce (2.4). 
Remark 2.5. Let $K, T, R$ be as in Corollary 2.4 and let $\tilde{X}, \tilde{Y}$ be the random vectors distributed uniformly on $K, T$ respectively. Corollary 2.4 states that

$$
\frac{1}{C R^{4}} \operatorname{Cov}(\tilde{X}) \leq \operatorname{Cov}(\tilde{Y}) \leq C R^{4} \operatorname{Cov}(\tilde{X})
$$

in the sense of symmetric matrices, where $\operatorname{Cov}(\tilde{X})$ is the covariance matrix of $\tilde{X}$. Furthermore, we do not have to assume that $\tilde{X}, \tilde{Y}$ are distributed uniformly in a convex body. The estimate (2.6) holds true whenever $\tilde{X}, \tilde{Y}$ have $\log$-concave densities $f_{\tilde{X}}, f_{\tilde{Y}}$ with

$$
R=\int_{\mathbb{R}^{n}}\left(\sup _{y \in \mathbb{R}^{n}} \sqrt{f_{\tilde{X}}(x+y) f_{\tilde{Y}}(x-y)}\right) d x .
$$

Next, for a measure $\mu$ and measurable sets $A, B$ with $0<\mu(A)<\infty$ define

$$
\left.\mu\right|_{A}(B)=\frac{\mu(A \cap B)}{\mu(A)} .
$$

Thus the probability measure $\left.\mu\right|_{A}$ is the conditioning of $\mu$ to the set $A$. Clearly, for a log-concave measure $\mu$ and an interval $I$, the measure $\left.\mu\right|_{I}$ remains log-concave.

Lemma 2.6. Let $\mu$ be a log-concave probability measure on $\mathbb{R}$. Then for any two intervals $J_{1} \subseteq J_{2} \subset \mathbb{R}$,

$$
\operatorname{Var}\left(\left.\mu\right|_{J_{1}}\right) \leq \operatorname{Var}\left(\left.\mu\right|_{J_{2}}\right)
$$

(the "intervals" may also be rays, or the entire line: Any convex set in $\mathbb{R}$ ).

Proof. It is enough to prove the lemma for $J_{1}, J_{2}$ being rays. Denote by $I$ the interior of the support of $\mu$, and by $\rho$ the density of $\mu$. Abbreviate $\Phi(t)=\mu((-\infty, t])$, $\mu_{t}=\left.\mu\right|_{(-\infty, t]}$ and set

$$
e(t)=\int_{\mathbb{R}} x d \mu_{t}(x), \quad v(t)=\operatorname{Var}\left(\mu_{t}\right)=\int_{\mathbb{R}} x^{2} d \mu_{t}(x)-e^{2}(t) \quad(t \in I) .
$$

Then for any $t \in I$,

$$
e^{\prime}(t)=\frac{\rho(t)}{\Phi(t)}(t-e(t)), \quad v^{\prime}(t)=\frac{\rho(t)}{\Phi(t)}\left((t-e(t))^{2}-v(t)\right) .
$$

To prove the lemma, it suffices to show that $v^{\prime}(t) \geq 0$ for any $t$, or equivalently, that

$$
\operatorname{Var}\left(\mu_{t}\right)-\left(t-\mathbb{E} \mu_{t}\right)^{2}=v(t)-(t-e(t))^{2} \leq 0 \quad \text { for all } t \in I .
$$

This is equivalent to demonstrating that for any log-concave random variable $X$ such that $X \geq 0$ almost surely, one has $\operatorname{Var}[X] \leq(\mathbb{E}[X])^{2}$. This follows immediately from Borell [5, Lemma 4.1], see also Lovász and Vempala [17, Lemma 5.3(c)]. 


\section{Deriving a stability estimate from the central limit theorem for convex sets}

In this section we prove Theorem 1.1. The main ingredient we use is the central limit theorem for convex sets, proven initially in [14]. It states that for any isotropic convex body $K \subset \mathbb{R}^{n}$, and for "most" subspaces of a small enough dimension, the marginal of $\mu_{K}$ is approximately Gaussian. Below we use a pointwise version of this theorem, proven in [9], which shows that there exists a subspace of dimension $n^{c}$, where $c>0$ is some universal constant, on which the marginals of both $K$ and $T$ are both approximately Gaussian density-wise. The Prékopa-Leindler inequality then implies that the marginal of $(K+T) / 2$ on the same subspace is pointwise greater than the supremum-convolution of the respective marginals of $K$ and $T$. Therefore, the density of the marginal of $(K+T) / 2$ must be greater than the supremum-convolution of two densities which are both approximately Gaussian, but typically have different covariances.

A second ingredient will be a calculation which shows that the integral of the supremum-convolution of two Gaussian densities whose covariance matrix is a multiple of the identity, becomes very large when their respective covariances are not close to one another. This will imply that when $\operatorname{Vol}_{n}((K+T) / 2)$ is not large, the covariance matrices of both marginals are roughly the same multiple of the identity. Therefore the inertia forms of $K$ and $T$ must have had roughly the same trace (the trace of the matrix will determine the multiple of the identity).

We write $G_{n, \ell}$ for the Grassmannian of all $\ell$-dimensional subspaces in $\mathbb{R}^{n}$, and $\sigma_{n, \ell}$ stands for the Haar probability measure on $G_{n, \ell}$. A random vector $X$ in $\mathbb{R}^{n}$ is centered if $\mathbb{E} X=0$ and is isotropic if its covariance matrix is the identity matrix. For a subspace $E \subseteq \mathbb{R}^{n}$ we write $\operatorname{Proj}_{E}$ for the orthogonal projection operator onto $E$ in $\mathbb{R}^{n}$. Furthermore, define $\gamma_{k, \alpha}(x)=\left(2 \pi \alpha^{2}\right)^{-k / 2} \exp \left(-\frac{|x|^{2}}{2 \alpha^{2}}\right)$ the centered Gaussian density in $\mathbb{R}^{k}$ with covariance $\alpha^{2}$, and abbreviate $\gamma_{k}(x)=\gamma_{k, 1}(x)$. The main result of [9] reads as follows:

Theorem 3.1. Let $X$ be a centered, isotropic random vector in $\mathbb{R}^{n}$ with a logconcave density. Let $1 \leq \ell \leq n^{c_{1}}$ be an integer. Then there exists a subset $\mathcal{E} \subseteq G_{n, \ell}$ with $\sigma_{n, \ell}(\mathcal{E}) \geq 1-C \exp \left(-n^{c_{2}}\right)$ such that for any $E \in \mathcal{E}$, the following holds: Denote by $f_{E}$ the log-concave density of the random vector $\operatorname{Proj}_{E}(X)$. Then,

$$
\left|\frac{f_{E}(x)}{\gamma_{\ell}(x)}-1\right| \leq \frac{C}{n^{c_{3}}}
$$

for all $x \in E$ with $|x| \leq n^{c_{4}}$. Here, $C, c_{1}, c_{2}, c_{3}, c_{4}>0$ are universal constants.

It can be seen directly from the proof in [9] that the constants in Theorem 3.1 may be selected to be $c_{1}, c_{2}, c_{3}=\frac{1}{30}, c_{4}=\frac{1}{60}, C=500$. Other constants would imply different universal constants in Theorem 1.1. We shall need the following elementary lemma: 
Lemma 3.2. For any $a>0$,

$$
\frac{1+a}{2 \sqrt{a}} \geq 1+c \cdot \min \left\{(\alpha-1)^{2}, 1\right\},
$$

for $\alpha=\sqrt{1 / a}$ and also for $\alpha=a$, where $c>0$ is a universal constant.

Proof. First we prove the lemma for $\alpha=a$. Note that for $0<a \leq 4$,

$$
\frac{1+a}{2 \sqrt{a}}=1+\frac{1-2 \sqrt{a}+a}{2 \sqrt{a}}=1+\frac{(\sqrt{a}-1)^{2}}{2 \sqrt{a}}=1+\frac{(a-1)^{2}}{2 \sqrt{a}(\sqrt{a}+1)} \geq 1+\frac{(a-1)^{2}}{12},
$$

while for $a>4$ we may write

$$
\frac{1+a}{2 \sqrt{a}}=1+\frac{(\sqrt{a}-1)^{2}}{2 \sqrt{a}} \geq 1+\frac{\sqrt{a}-1}{2 \sqrt{a}} \geq 1+\frac{\sqrt{a} / 2}{2 \sqrt{a}}=1+\frac{1}{4} .
$$

The case where $\alpha=\sqrt{1 / a}$ follows as $\min \left\{(\sqrt{1 / a}-1)^{2}, 1\right\} \leq 10 \min \left\{(a-1)^{2}, 1\right\}$.

The following lemma is the second ingredient in our proof of Theorem 1.1 described above. The essence of the lemma is that the integral of the supremumconvolution of two spherically-symmetric Gaussian densities must be quite large when the covariances are not close to each other.

Lemma 3.3. Let $k \in \mathbb{N}$ and $A, B, \alpha>0$. Let $f, g, h: \mathbb{R}^{k} \rightarrow[0, \infty)$ satisfy

$$
h(x) \geq \sup _{y \in \mathbb{R}^{k}} \sqrt{f(x-y) g(x+y)}, \quad \forall x \in \mathbb{R}^{k}
$$

and suppose that,

$$
f(x) \geq A \gamma_{k, 1}(x)
$$

whenever $|x| \leq 10 \sqrt{k}$, and that

$$
g(x) \geq B \gamma_{k, \alpha}(x),
$$

whenever $|x| \leq 10 \alpha \sqrt{k}$. Assume that $h$ is measurable. Then,

$$
\int_{\mathbb{R}^{k}} h(x) d x \geq \frac{1}{2} \sqrt{A B}\left(1+c \cdot \min \left\{(\alpha-1)^{2}, 1\right\}\right)^{k / 4},
$$

where $c>0$ is a universal constant. 
Proof. By homogeneity, we may assume that $A=B=1$. Denote $a=1 / \alpha^{2}$. Fix a unit vector $\theta \in \mathbb{R}^{n}$ and $t>0$. Then for any $s \in \mathbb{R}$ with $|s+t| \leq 10 \sqrt{k}$ and $|s-t| \leq 10 \alpha \sqrt{k}$

$$
\begin{aligned}
h(t \theta) & \geq \sqrt{f((t+s) \theta) g((t-s) \theta)} \\
& \geq\left(\frac{\sqrt{a}}{2 \pi}\right)^{k / 2} \exp \left(-\frac{1}{4}\left((t+s)^{2}+a(t-s)^{2}\right)\right) .
\end{aligned}
$$

We would like to find $s$ which maximizes the right-hand side in (3.3). We select $s=t(a-1) /(a+1)$ and verify that when $|t|<5 \sqrt{(1+a) k / a}$ we have $|s+t| \leq$ $10 \sqrt{k}$ and $|s-t| \leq 10 \alpha \sqrt{k}$. We conclude that for any $|t|<5 \sqrt{(1+a) k / a}$,

$$
h(t \theta) \geq\left(\frac{\sqrt{a}}{2 \pi}\right)^{k / 2} \exp \left(-t^{2} a /(1+a)\right) \text {. }
$$

Consequently,

$$
\begin{aligned}
\int_{\mathbb{R}^{k}} h(x) d x & \geq\left(\frac{\sqrt{a}}{2 \pi}\right)^{k / 2} \int_{5 \sqrt{(1+a) k / a} B_{2}^{k}} \exp \left(-\frac{a|x|^{2}}{1+a}\right) d x \\
& =\left(\frac{1+a}{4 \pi \sqrt{a}}\right)^{k / 2} \int_{\sqrt{50 k} B_{2}^{k}} \exp \left(-\frac{|x|^{2}}{2}\right) d x \geq \frac{1}{2}\left(\frac{1+a}{2 \sqrt{a}}\right)^{k / 2},
\end{aligned}
$$

where $B_{2}^{k}=\left\{x \in \mathbb{R}^{k} ;|x| \leq 1\right\}$, and where we utilized the fact that

$$
\mathbb{P}\left(|Z|^{2} \geq 50 k\right) \leq \mathbb{E}|Z|^{2} /(50 k)=\frac{1}{50}<1 / 2
$$

when $Z$ is a standard Gaussian in $\mathbb{R}^{k}$. All that remains to do is to apply Lemma 3.2.

The following lemma combines Theorem 3.1 with the estimate we have just proved. For a probability density $g$ on $\mathbb{R}^{n}$ we write $\operatorname{Cov}(g)$ for the covariance matrix of the random vector with density $g$. We similarly define $\operatorname{Cov}(\mu)$ for a probability measure $\mu$ on $\mathbb{R}^{n}$.

Lemma 3.4. Let $f, g$ be log-concave probability densities on $\mathbb{R}^{n}$ such that $f$ is isotropic. Let $\left\{\lambda_{i}\right\}_{i=1}^{n}$ be the eigenvalues of $\operatorname{Cov}(g)$, repeated according to their multiplicity. Denote

$$
R=\int_{\mathbb{R}^{n}} \sup _{y \in \mathbb{R}^{n}} \sqrt{f(x+y) g(x-y)} d x .
$$

Then, for $0<\delta<1$,

$$
\#\left\{i ;\left|\lambda_{i}-1\right| \geq \delta\right\} \leq C\left(\frac{\log (2 R)}{\delta}\right)^{C_{1}}
$$

for some universal constants $C, C_{1}>1$. 
Proof. Clearly, we may assume that the sequence $\left\{\lambda_{i}\right\}$ is non-decreasing. Translating $g$, we may assume that the barycenter of $g$ is at the origin. Let $X$ and $Y$ be random vectors that are distributed according to the laws $f, g$, respectively. Fix $0<\delta<1$. Consider the subspace $E$ spanned by $\left\{e_{i} ; \lambda_{i}-1 \geq \delta\right\}$, where $\left\{e_{i}\right\}$ is an orthonormal basis of eigenvectors corresponding the the eigenvalues $\left\{\lambda_{i}\right\}$. Denote $d=\operatorname{dim} E$ and assume that $d \geq 2$. Since the $\lambda_{i}$ 's are in increasing order, the subspace $E$ has the form,

$$
E=\operatorname{span}\left\{e_{i}, i \geq i_{0}\right\}
$$

for some $1 \leq i_{0} \leq n$. Write $j_{0}=\left\lfloor\frac{n-i_{0}}{2}\right\rfloor$ and $V^{2}=\lambda_{i_{0}+j_{0}}$. Now, fix $1 \leq j \leq j_{0}$. Define,

$$
v_{j}(\theta)=\theta e_{i_{0}+j_{0}+j}+\sqrt{1-\theta^{2}} e_{i_{0}+j_{0}-j} .
$$

Inspect the function $f(\theta)=\left\langle\operatorname{Cov}(g) v_{j}(\theta), v_{j}(\theta)\right\rangle$. We have $f(0)=\lambda_{i_{0}+j_{0}-j} \leq$ $V^{2}$ and $f(1)=\lambda_{i_{0}+j_{0}+j} \geq V^{2}$. By continuity, there exists a certain $0 \leq \theta_{j} \leq 1$ for which

$$
\left\langle\operatorname{Cov}(g) v_{j}\left(\theta_{j}\right), v_{j}\left(\theta_{j}\right)\right\rangle=V^{2} .
$$

Denote

$$
F=\operatorname{span}\left\{v_{j}\left(\theta_{j}\right) \mid 1 \leq j \leq j_{0}\right\} .
$$

Equation (3.4) and the fact that $e_{1}, \ldots, e_{n}$ are orthonormal eigenvectors imply that for every $v \in F$, one has $\langle\operatorname{Cov}(g) v, v\rangle=V^{2}$. Moreover, $\operatorname{dim} F=j_{0} \geq \frac{1}{2} d-1$. We now apply Theorem 3.1 which claims that if $d \geq C$, then there exists a subspace $G \subset F$ with $\operatorname{dim} G=\left\lfloor d^{1 / 40}\right\rfloor$ such that

$$
\tilde{f}(x) \geq \frac{1}{2} \gamma_{k, 1}(x), \quad \tilde{g}(y) \geq \frac{1}{2} \gamma_{k, V}(y)
$$

for all $x$ with $|x| \leq 10 d^{1 / 80}$ and for all $|y| \leq 10 V d^{1 / 80}$, where $\tilde{f}$ and $\tilde{g}$ are the densities of $\operatorname{Proj}_{G}(X), \operatorname{Proj}_{G}(Y)$ respectively. Next, we use Lemma 3.3 to attain

$$
\int_{G} \sup _{y \in G} \sqrt{\tilde{f}(x-y) \tilde{g}(x+y)} d x \geq \frac{1}{4}\left(1+c \cdot \min \left\{(V-1)^{2}, 1\right\}\right)^{\operatorname{dim} G / 4} .
$$

On the other hand, we may use the Prekopá-Leindler inequality as in (2.5) above, and deduce that

$$
\int_{G} \sup _{y \in G} \sqrt{\tilde{f}(x-y) \tilde{g}(x+y)} d x \leq R .
$$

Consequently, under the assumption that $d \geq C$,

$$
\min \left\{(V-1)^{2}, 1\right\} \leq C \log (2 R) / \operatorname{dim}(G) .
$$

Since $V \geq \sqrt{1+\delta} \geq 1+\delta / 3$, we conclude

$$
\#\left\{i ; \lambda_{i}-1 \geq \delta\right\} \leq C\left(\frac{\log (2 R)}{\delta}\right)^{C_{1}} .
$$


By repeating the argument, with the subspace $\left\{e_{i} ; \lambda_{i}-1 \leq-\delta\right\}$ replacing the subspace $E$, we conclude the proof.

Proof of Theorem 1.1. By applying affine transformations to both $K$ and $T$, we can assume that both bodies have the origin as their barycenter, and that $p_{K}(x)=|x|^{2}$ while $p_{T}(x)=\sum_{i} x_{i}^{2} / \lambda_{i}$. By Lemma 3.4 ,

$$
\#\left\{i ;\left|\lambda_{i}-1\right| \geq \delta\right\} \leq C\left(\frac{\log (2 R)}{\delta}\right)^{C_{1}},
$$

for any $0<\delta<1$. Since $\lambda_{i} \leq C R^{4}$ for all $i$, as follows from Corollary 2.4, then

$$
\frac{1}{n} \sum_{i=1}^{n}\left(\lambda_{i}-1\right)^{2} \leq \frac{C}{n} \int_{0}^{1} \min \left\{n,\left(\frac{\log (2 R)}{\delta}\right)^{C_{1}}\right\} d \delta+\frac{\tilde{C}(\log (2 R))^{C_{1}} R^{4}}{n} \leq C \frac{R^{\alpha_{2}}}{n^{\alpha_{1}}}
$$

where $C, \alpha_{1}, \alpha_{2}>0$ are universal constants. To obtain (1.6), note that

$$
\left|\frac{\int_{T} p_{K}\left(x-b_{T}\right) d \mu_{T}(x)}{\int_{K} p_{K}\left(x-b_{K}\right) d \mu_{K}(x)}-1\right|=\frac{1}{n}\left|\sum_{i=1}^{n}\left(\lambda_{i}-1\right)\right| \leq \sqrt{\frac{1}{n} \sum_{i=1}^{n}\left(\lambda_{i}-1\right)^{2}} .
$$

Remark 3.5. When $K$ in Theorem 1.1 is isotropic, we actually prove in (3.7) that

$$
\left\|\operatorname{Cov}\left(\mu_{K}\right)-\operatorname{Cov}\left(\mu_{T}\right)\right\|_{H S}^{2} \leq C R^{\alpha_{2}} n^{1-\alpha_{1}},
$$

where $\|A\|_{H S}^{2}=\operatorname{Trace}\left(A^{t} A\right)$ is the square of the Hilbert-Schmidt norm of the matrix $A$.

\section{Obtaining stability estimates using a transportation argument}

The goal of this section is to prove Theorem 1.2 and to obtain some quantitative estimates for the exponents from Theorem 1.1. We begin with several core definitions which will be used in the proof. For two measurable functions $f, g: \mathbb{R}^{n} \rightarrow[0, \infty)$, denote by $H_{\lambda}(f, g)$ the supremum-convolution of the two functions, hence,

$$
H_{\lambda}(f, g)(x):=\sup _{y \in \mathbb{R}^{n}} f^{1-\lambda}(x+\lambda y) g^{\lambda}(x-(1-\lambda) y) .
$$

The function

$$
(\lambda, x) \mapsto H_{\lambda}(f, g)(x)
$$


is log-concave in $[0,1] \times \mathbb{R}^{n}$. We define

$$
K_{\lambda}(f, g)=\int_{\mathbb{R}^{n}} H_{\lambda}(f, g)(x) d x
$$

the integral over a subspace, and

$$
K(f, g)=\int_{0}^{1} K_{\lambda}(f, g) d \lambda
$$

the entire integral. Next, we write

$$
b(f, g)=\frac{1}{K(f, g)} \int_{\mathbb{R}^{n}} \int_{0}^{1} x H_{\lambda}(f, g)(x) d \lambda d x,
$$

the barycenter of $\int_{0}^{1} H_{\lambda}(f, g)(x) d \lambda$. For $x \in \mathbb{R}^{n}$ we write $x \otimes x=\left(x_{i} x_{j}\right)_{i, j=1, \ldots, n}$, an $n \times n$ matrix. Set

$$
D(f, g)=\frac{1}{K(f, g)} \int_{\mathbb{R}^{n}} \int_{0}^{1}(x \otimes x) H_{\lambda}(f, g)(x+b(f, g)) d \lambda d x,
$$

the covariance matrix. Finally, we normalize this density by defining

$$
L(f, g)(\lambda, x)=\frac{1}{K(f, g)} \sqrt{\operatorname{det} D(f, g)} \cdot H_{\lambda}(f, g)\left(D^{1 / 2} x+b(f, g)\right)
$$

and

$$
l(f, g)(x)=\int_{0}^{1} L(f, g)(\lambda, x) d \lambda,
$$

the marginal of $L(f, g)$ with respect to the axis $\lambda$. Note that by the Prékopa-Leindler inequality, $l(f, g)$ is an isotropic log-concave probability density in $\mathbb{R}^{n}$.

The results of this section rely on the so-called Brenier map between two given $\log$-concave measures. Given two smooth log-concave probability densities $f, g$ on $\mathbb{R}^{n}$, one may consider the Monge-Ampère equation,

$$
\operatorname{det}(\operatorname{Hess} \varphi)=\frac{g \circ \nabla \varphi}{f} .
$$

A theorem of Brenier asserts that a convex solution to the above equation on the domain $\operatorname{Supp}(f)=\{x ; f(x)>0\}$ exists. The regularity theory developed by Caffarelli implies that the convex function $\varphi$ is smooth. For precise definitions and properties, see [22]. The map $F=\nabla \varphi$ pushes forward the measure whose density is $f$ to the measure whose density is $g$, and is referred to as the Brenier map between the two measures. The matrix $\nabla F(x)$ is positive-definite since it has a positive determinant and it is the Hessian matrix of a convex function. 
Remark 4.1. The Knothe map, used in Section 6, is in some sense a limiting case of the Brenier map. See [7].

The following lemma contains the central idea of this section.

Lemma 4.2. Let $f, g$ be log-concave probability densities in $\mathbb{R}^{n}$. Denote $K=$ $K(f, g)$. Let $x \rightarrow F(x)$ be the Brenier map pushing forward the measure whose density is $f$ to the measure whose density is $g$. Suppose that $X$ is a random vector distributed according to the law $l(f, g)$ in $\mathbb{R}^{n}$. Then,

$\operatorname{Var}\left[|X|^{2}\right] \geq \frac{1}{K(f, g)} \int_{\mathbb{R}^{n}} f(x) \operatorname{Var}\left[\left|D^{-1 / 2}((1-\Lambda) x+\Lambda F(x)-b(f, g))\right|^{2}\right] d x$

where $D=D(f, g)$ and $\Lambda$ is a random variable distributed uniformly in $[0,1]$.

Proof. By a standard approximation argument we may assume that $f$ and $g$ are sufficiently smooth. Denote $D=D(f, g)$ and $L(\lambda, x)=L(f, g)(\lambda, x)$. Furthermore, define,

$$
\tilde{f}(x)=\sqrt{\operatorname{det} D} \cdot f\left(D^{1 / 2} x+b(f, g)\right), \quad \tilde{g}(x)=\sqrt{\operatorname{det} D} \cdot g\left(D^{1 / 2} x+b(f, g)\right)
$$

so that $\tilde{f}(x)=K(f, g) L(0, x)$ and $\tilde{g}(x)=K(f, g) L(1, x)$. Denote

$$
\tilde{F}(x)=D^{-1 / 2}\left(F\left(D^{1 / 2} x+b(f, g)\right)-b(f, g)\right) .
$$

Then $\tilde{F}$ pushes forward the measure whose density is $\tilde{f}$ to the measure whose density is $\tilde{g}$. Next, define

$$
M(\lambda, x)=\left(M_{1}(\lambda, x), M_{2}(\lambda, x)\right)=(\lambda,(1-\lambda) x+\lambda \tilde{F}(x)) .
$$

By elementary properties of the Brenier map, $M$ is a one-to-one map from $[0,1] \times$ $\operatorname{Supp}(\tilde{f})$ to $\operatorname{Supp}(L)$. Define a density,

$$
q(\lambda, x)=\frac{\tilde{f}(x)^{(1-\lambda)} \tilde{g}(\tilde{F}(x))^{\lambda}}{K(f, g)}=L(0, x)^{1-\lambda} L(1, \tilde{F}(x))^{\lambda} .
$$

Using the fact that $L$ is log-concave, we obtain

$$
q(\lambda, x) \leq L(M(\lambda, x)), \quad \forall \lambda \in[0,1], x \in \operatorname{Supp}(\tilde{f})
$$

A simple calculation shows that the Jacobian of $M(\lambda, x)$ is

$$
J(\lambda, x)=\operatorname{det}((1-\lambda) I d+\lambda \nabla \tilde{F}(x)) .
$$

Recall that $\operatorname{det}(\nabla \tilde{F}(x))=\frac{\tilde{f}(x)}{\tilde{g}(\tilde{F}(x))}$. Furthermore, the matrix $\nabla \tilde{F}(x)$ is diagonalizable with positive eigenvalues, since it is conjugate to the matrix $\nabla F\left(D^{1 / 2} x+\right.$ 
$b(f, g))$ which is a positive-definite matrix. By the arithmetic/geometric means inequality,

$$
J(\lambda, x) \geq \operatorname{det}(\nabla \tilde{F}(x))^{\lambda}=\left(\frac{\tilde{f}(x)}{\tilde{g}(\tilde{F}(x))}\right)^{\lambda}
$$

Therefore,

$$
J(\lambda, x) q(\lambda, x) \geq \frac{\tilde{f}(x)}{K(f, g)}, \quad \forall \lambda \in[0,1], x \in \mathbb{R}^{n} .
$$

By changing variables using $M^{-1}$ and applying (4.4) and (4.5), we calculate

$$
\begin{aligned}
\operatorname{Var}\left[|X|^{2}\right] & =\int_{\mathbb{R}^{n}} \int_{[0,1]}\left(|x|^{2}-\int_{\mathbb{R}^{n}} \int_{[0,1]}|y|^{2} L(\theta, y) d \theta d y\right)^{2} L(\lambda, x) d \lambda d x \\
& \geq \int_{\mathbb{R}^{n}} \int_{[0,1]}\left(\left|M_{2}(\lambda, x)\right|^{2}-\int_{\mathbb{R}^{n}} \int_{[0,1]}|y|^{2} L(\theta, y) d \theta d y\right)^{2} J(\lambda, x) q(\lambda, x) d \lambda d x \\
& \geq \int_{\mathbb{R}^{n}} \frac{\tilde{f}(x)}{K(f, g)}\left(\int_{[0,1]}\left(\left|M_{2}(\lambda, x)\right|^{2}-\int_{\mathbb{R}^{n}} \int_{[0,1]}|y|^{2} L(\theta, y) d \theta d y\right)^{2} d \lambda\right) d x \\
& \geq \int_{\mathbb{R}^{n}} \frac{\tilde{f}(x)}{K(f, g)}\left(\int_{[0,1]}\left(\left|M_{2}(\lambda, x)\right|^{2}-\int_{[0,1]}\left|M_{2}(\theta, x)\right|^{2} d \theta\right)^{2} d \lambda\right) d x \\
& =\int_{\mathbb{R}^{n}} \frac{\tilde{f}(x)}{K(f, g)} \operatorname{Var}\left[|(1-\Lambda) x+\Lambda \tilde{F}(x)|^{2}\right] d x .
\end{aligned}
$$

Applying the change of variables $x \rightarrow D^{-1 / 2}(x-b(f, g))$ completes the proof.

By the definition of the thin-shell parameter $\sigma_{n}$ from [10], for any isotropic random vector $X$ in $\mathbb{R}^{n}$ with a log-concave density, one has,

$$
\operatorname{Var}\left[|X|^{2}\right] \leq C n \sigma_{n}^{2}
$$

Combining this with the above lemma yields

$$
\int_{\mathbb{R}^{n}} f(x) \operatorname{Var}\left[\left|D(f, g)^{-1 / 2}((1-\Lambda) x+\Lambda F(x)-b(f, g))\right|^{2}\right] d x \leq C K(f, g) n \sigma_{n}^{2} .
$$

For $x, y \in \mathbb{R}^{n}$, define,

$$
v(x, y)=\operatorname{Var}\left[|\Lambda x+(1-\Lambda) y|^{2}\right]
$$

In view of (4.7), we would like to have a lower bound for $v(x, y)$ in terms of $|x|^{2}-$ $|y|^{2}$ and in terms of $|x-y|$. The following lemma serves this purpose. 
Lemma 4.3. There exist universal constants $C_{1}, C_{2}>0$, such that for all $x, y \in$ $\mathbb{R}^{n}$

$$
v(x, y)=C_{1}\left(|x|^{2}-|y|^{2}\right)^{2}+C_{2}|x-y|^{4} .
$$

Proof. Define

$$
f(\lambda)=|\lambda x+(1-\lambda) y|^{2}, \quad g(\lambda)=\lambda|x|^{2}+(1-\lambda)|y|^{2},
$$

and $h(\lambda)=f(\lambda)-g(\lambda)$. Then $h(1-\lambda)=h(\lambda)$ hence $\operatorname{COV}(g(\Lambda), h(\Lambda))=0$. Consequently,

$$
\operatorname{Var}[f(\Lambda)]=\operatorname{Var}[h(\Lambda)]+\operatorname{Var}[g(\Lambda)] .
$$

It is easy to verify that

$$
\operatorname{Var}[g(\Lambda)]=\left(|x|^{2}-|y|^{2}\right)^{2} \operatorname{Var}(\Lambda)=C_{1}\left(|x|^{2}-|y|^{2}\right)^{2} .
$$

Next, using the parallelogram law,

$$
h(\lambda)=-\lambda(1-\lambda)|x-y|^{2} .
$$

Consequently,

$$
\operatorname{Var}[h(\Lambda)]=|x-y|^{4} \operatorname{Var}[\Lambda(1-\Lambda)]=C_{2}|x-y|^{4} .
$$

Combining (4.9), (4.10) and (4.11) completes the proof.

Proof of Theorem 1.2. Write $b=b(f, g)$ and $D=D(f, g)$. Substituting the result of Lemma 4.3 into (4.7) yields

$$
\begin{aligned}
\int_{\mathbb{R}^{n}} f(x) & \left(\left(\left|D^{-1 / 2}(x-b)\right|^{2}-\left|D^{-1 / 2}(F(x)-b)\right|^{2}\right)^{2}+\left|D^{-1 / 2}(x-F(x))\right|^{4}\right) d x \\
& \leq C K(f, g) n \sigma_{n}^{2} .
\end{aligned}
$$

Let $X, Y$ be the random vectors whose densities are $f, g$ respectively. By the definition of the transportation distance,

$$
W_{2}^{2}\left(D^{-1 / 2} X, D^{-1 / 2} Y\right) \leq \int_{\mathbb{R}^{n}} f(x)\left|D^{-1 / 2}(x-F(x))\right|^{2} d x,
$$

where the transportation distance between random vectors is defined to be the distance between the corresponding distribution measures. The fact that $f$ and $g$ have barycenters at the origin implies

$$
\mathbb{E}\left[\left\langle D^{-1 / 2} X, D^{-1 / 2} d\right\rangle\right]=\mathbb{E}\left[\left\langle D^{-1 / 2} Y, D^{-1 / 2} d\right\rangle\right]=0,
$$


and consequently

$$
\begin{gathered}
\int_{\mathbb{R}^{n}} f(x)\left(\left|D^{-1 / 2}(x-d)\right|^{2}-\left|D^{-1 / 2}(F(x)-d)\right|^{2}\right) d x \\
=\operatorname{Tr}\left(\operatorname{Cov}\left(D^{-1 / 2} X\right)-\operatorname{Cov}\left(D^{-1 / 2} Y\right)\right) .
\end{gathered}
$$

The Cauchy-Schwartz inequality together with (4.12), (4.13) and (4.14) yield,

$$
W_{2}(\tilde{X}, \tilde{Y})^{4}+[\operatorname{Tr}(\operatorname{Cov}(\tilde{X})-\operatorname{Cov}(\tilde{Y}))]^{2} \leq C n K(f, g) \sigma_{n}^{2}
$$

where $\tilde{X}=D^{-1 / 2} X$ and $\tilde{Y}=D^{-1 / 2} Y$. Consequently,

$$
W_{2}(X, Y)^{2} \leq C \sqrt{n K(f, g)} \sigma_{n}\|D\|_{O P}
$$

where $\|D\|_{O P}=\sup _{0 \neq x}|D(x)| /|x|$ is the operator norm of $D$. From the remark to Corollary 2.4 we conclude that

$$
\|D\|_{O P} \leq C K_{1 / 2}(f, g)^{4}
$$

The function $\lambda \mapsto K_{\lambda}(f, g)$ is log-concave and it is bounded from below by one, according to the Prékopa-Leindler inequality. Therefore,

$$
K_{1 / 2}(f, g) \geq \sqrt{\sup _{\lambda \in(0,1)} K_{\lambda}(f, g)} \geq \sqrt{K(f, g)},
$$

and (1.9) is proven.

The rest of this section aims at a better understanding of the exponents in Theorem 1.1. The next lemma exploits the second summand in our basic estimate (4.15).

Lemma 4.4. Let $f$, $g$ be log-concave probability densities on $\mathbb{R}^{n}$ whose barycenters are at the origin. Suppose that $f$ is isotropic. Then there exists a universal constant $c_{1}>0$ such that whenever $K_{1 / 2}(f, g) \leq \exp \left(n^{c_{1}}\right)$, there exist two unit vectors $\theta_{1}, \theta_{2} \in \mathbb{R}^{n}$ with

$$
\left\langle\operatorname{Cov}(g) \theta_{1}, \theta_{1}\right\rangle \leq 1+C \sigma_{n} \sqrt{\frac{K(f, g)}{n}}
$$

and

$$
\left\langle\operatorname{Cov}(g) \theta_{2}, \theta_{2}\right\rangle \geq 1-C \sigma_{n} \sqrt{\frac{K(f, g)}{n}} .
$$

Here, $C>0$ is some universal constant. 
Proof. We use the notation of the proof of Theorem 1.2. In order to establish (4.16), we fix $\alpha>0$, and assume that

$$
\langle\operatorname{Cov}(g) \theta, \theta\rangle>1+\alpha \sigma_{n} \sqrt{\frac{K(f, g)}{n}}, \quad \forall \theta \in \mathbb{S}^{n-1},
$$

where $\mathbb{S}^{n-1}=\left\{x \in \mathbb{R}^{n}:|x|=1\right\}$. Our goal is to show that necessarily $\alpha \leq C$. Noting that $\operatorname{Cov}(\tilde{X})=D^{-1}$ we have

$$
\left\langle\operatorname{Cov}(\tilde{X})^{-1 / 2} \operatorname{Cov}(\tilde{Y}) \operatorname{Cov}(\tilde{X})^{-1 / 2} \theta, \theta\right\rangle-1>\alpha \sigma_{n} \sqrt{\frac{K(f, g)}{n}}, \quad \forall \theta \in \mathbb{S}^{n-1},
$$

where $\tilde{X}$ and $\tilde{Y}$ are as in the proof of Theorem 1.2. The last inequality implies,

$$
\frac{\operatorname{Tr}(\operatorname{Cov}(\tilde{Y}))}{\operatorname{Tr}(\operatorname{Cov}(\tilde{X}))}-1>\alpha \sigma_{n} \sqrt{\frac{K(f, g)}{n}} .
$$

Consequently, in order to establish (4.16), it suffices to show that for some universal constant $C>0$,

$$
|\operatorname{Tr}(\operatorname{Cov}(\tilde{Y}))-\operatorname{Tr}(\operatorname{Cov}(\tilde{X}))| \leq C \operatorname{Tr}(\operatorname{Cov}(\tilde{X})) \sigma_{n} \sqrt{\frac{K(f, g)}{n}} .
$$

In view of (4.15), the last inequality will be concluded if we only manage to show

$$
\operatorname{Tr}(\operatorname{Cov}(\tilde{X}))=\operatorname{Tr}\left(D^{-1}\right) \geq \frac{n}{2} .
$$

The above fact follows from an application of Lemma 3.4 with $\delta=1 / 2$ and from the assumption that $K_{1 / 2}(f, g) \leq \exp \left(n^{c_{1}}\right)$. Equation (4.16) is established, and the proof of (4.17) is analogous. The proof of the lemma is thus complete.

Next, define

$$
\kappa=\limsup _{n \rightarrow \infty} \frac{\log \sigma_{n}}{\log n}, \quad \tau_{n}=\max \left\{1, \max _{1 \leq j \leq n} \frac{\sigma_{j}}{j^{\kappa}}\right\},
$$

so that $\sigma_{n} \leq \tau_{n} n^{\kappa}$. Note that the thin-shell conjecture implies that $\kappa=0$ and $\tau_{n}<C$. We apply the estimate from the previous lemma for various marginals of our $n$-dimensional measures, and obtain:

Lemma 4.5. Let $f$, $g$ be log-concave probability densities in $\mathbb{R}^{n}$ whose barycenter is at the origin. Suppose that $f$ is isotropic. Define $R=K_{1 / 2}(f, g)$ and denote by $\left\{\lambda_{i}\right\}$ the eigenvalues of $\operatorname{Cov}(g)$, repeated according to their multiplicity. Assume that the sequence $\left\{\left|\lambda_{i}-1\right|\right\}$ is non-increasing. Then, one has

$$
\left|\lambda_{i}-1\right| \leq C R^{4}, \quad \forall 1 \leq i \leq n
$$

and

$$
\left|\lambda_{i}-1\right| \leq C R \tau_{n} i^{\kappa-\frac{1}{2}}, \quad \forall(\log (2 R))^{C_{1}} \leq i \leq n
$$

where $C, C_{1}>0$ are some universal constants. 
Proof. The bound (4.20) follows directly from the remark to Corollary 2.4. In order to establish (4.21), denote by $\left\{e_{i}\right\}$ the orthonormal basis of eigenvectors corresponding to the eigenvalues $\left\{\lambda_{i}\right\}$. Define

$$
E_{1}=\operatorname{sp}\left\{e_{j} ; 1 \leq j \leq i, \lambda_{j} \geq 1\right\}, \quad E_{2}=\operatorname{sp}\left\{e_{j} ; 1 \leq j \leq i, \lambda_{j} \leq 1\right\}
$$

Let $E$ be the subspace with the larger dimension among these two subspaces. Then $k=\operatorname{dim} E \geq i / 2$. Denote by $i_{0}$ the maximal $j$ for which $e_{j} \in E$. Then $k \leq i_{0} \leq i$. According to our assumption, $\operatorname{dim}(E) \geq(\log (2 R))^{C_{1}} / 2$, and hence we may apply Lemma 4.4 in the subspace $E$. Denote by $f_{E}$ and $g_{E}$ the marginals of $f$ and $g$ to the subspace $E$. Using (4.16) and (4.17) for $f_{E}$ and $g_{E}$ we obtain

$$
\left|\lambda_{i}-1\right| \leq\left|\lambda_{i_{0}}-1\right| \leq C \sigma_{k} \sqrt{\frac{K(f, g)}{k}} \leq C^{\prime} R \tau_{k} i^{\kappa-\frac{1}{2}} \leq C^{\prime} R \tau_{n} i^{\kappa-\frac{1}{2}}
$$

where we used the fact that $K(f, g) \leq K_{1 / 2}(f, g)^{2}=R^{2}$ as well as the PrékopaLeindler inequality which implies that $K_{\lambda}\left(f_{E}, g_{E}\right) \leq K_{\lambda}(f, g)$ for any $\lambda \in(0,1)$.

The next theorem demonstrates that the exponent $\alpha_{1}$ in Theorem 1.1 may be made arbitrarily close to $1 / 2-\kappa$, thus complementing the inequality (1.7) which goes in the opposite direction. This provides yet another piece of evidence for the close relationship between the thin shell problem and the stability of the BrunnMinkowski inequality in high dimensions.

Theorem 4.6. Let $K, T \subset \mathbb{R}^{n}$ be convex bodies and let $R \geq 1$. Assume that $K$ is isotropic, that the barycenter of $T$ is at the origin and that

$$
\operatorname{Vol}_{n}\left(\frac{K+T}{2}\right) \leq C R \sqrt{\operatorname{Vol}_{n}(K) \operatorname{Vol}_{n}(T)}
$$

Then,

$$
\left\|\operatorname{Cov}\left(\mu_{T}\right)-I d\right\|_{H S} \leq C\left(R^{5}+\tau_{n} R \max \left(\sqrt{\log n}, n^{\kappa}\right)\right),
$$

where Id is the identity matrix. Consequently,

$$
\begin{aligned}
\left|\frac{\int_{T}|x|^{2} d \mu_{T}(x)}{\int_{K}|x|^{2} d \mu_{K}(x)}-1\right| & \leq \frac{\left\|\operatorname{Cov}\left(\mu_{T}\right)-I d\right\|_{H S}}{\sqrt{n}} \\
& \leq C \frac{R^{5}+\tau_{n} R \max \left(\sqrt{\log n}, n^{\kappa}\right)}{\sqrt{n}} .
\end{aligned}
$$

Here, $C>0$ is a universal constant. 
Proof. We may clearly assume that $\operatorname{Cov}\left(\mu_{T}\right)$ is a diagonal matrix whose diagonal is $\lambda_{1}, \ldots, \lambda_{n}$, where the sequence $\left\{\left|\lambda_{i}-1\right|\right\}$ is non-increasing. Since our measures are log-concave, then we may use Lemma 4.5 and calculate

$$
\begin{aligned}
\sum_{i=1}^{n}\left|\lambda_{i}-1\right|^{2} & \leq C R^{8}(\log (2 R))^{C_{1}}+C R^{2} \tau_{n}^{2} \sum_{i=1}^{n} i^{2 \kappa-1} \\
& \leq \tilde{C} R^{9}+C R^{2} \tau_{n}^{2}\left(1+\int_{1}^{n} s^{2 \kappa-1} d s\right) \\
& \leq C^{\prime}\left(R^{9}+\tau_{n}^{2} R^{2} \max \left(\log n, n^{2 \kappa}\right)\right)
\end{aligned}
$$

The bound (4.24) follows. In order to deduce (4.25) from (4.24), argue as in (3.8) above. The proof is complete.

\section{Transportation in one dimension}

In this section we recall some basic definitions concerning transportation of onedimensional measures. For a Borel measure $\mu$ in $\mathbb{R}^{n}$ we write $\overline{\operatorname{Supp}(\mu)}$ for the set of all points $x \in \mathbb{R}^{n}$ such that all of the neighborhoods of $x$ have positive $\mu$ measure. The support of $\mu$, denoted by $\operatorname{Supp}(\mu)$, is defined in this paper to be the interior of $\overline{\operatorname{Supp}(\mu)}$. Suppose that $\mu_{1}$ and $\mu_{2}$ are Borel probability measures on the real line, with continuous densities $\rho_{1}$ and $\rho_{2}$, respectively. We further assume that the $\operatorname{Supp}\left(\mu_{2}\right)$ is connected and that $\rho_{2}$ does not vanish in its support. For $t \in \mathbb{R}$ set

$$
\Phi_{j}(t)=\mu_{j}((-\infty, t]) \quad(j=1,2) .
$$

For $j=1,2$, the map $\Phi_{j}^{-1}$ pushes forward the uniform measure on $[0,1]$ to $\mu_{j}$. The monotone transportation map between $\mu_{1}$ and $\mu_{2}$ is the continuous, non-decreasing function

$$
F(t)=\Phi_{2}^{-1}\left(\Phi_{1}(t)\right)
$$

defined for $t \in \operatorname{Supp}\left(\mu_{1}\right)$. Observe that

$$
F_{*}\left(\mu_{1}\right)=\mu_{2}
$$

Furthermore, $F$ is differentiable in $\operatorname{Supp}\left(\mu_{1}\right)$ and

$$
\rho_{1}(t)=F^{\prime}(t) \rho_{2}(F(t)) \quad \text { for } t \in \operatorname{Supp}\left(\mu_{1}\right) .
$$

Additionally, it is well-known (see, e.g., Villani's book [22]) that

$$
W_{2}\left(\mu_{1}, \mu_{2}\right)=\sqrt{\int_{\mathbb{R}}|F(x)-x|^{2} d \mu_{1}(x)} .
$$

A probability measure on $\mathbb{R}$ is said to be even if $\mu(A)=\mu(-A)$ for any measurable $A \subset \mathbb{R}$, where $-A=\{-x: x \in A\}$. 
Proposition 5.1. Suppose that $\mu_{1}$ and $\mu_{2}$ are even, log-concave probability measures on $\mathbb{R}$. Denote $\sigma=\sqrt{\operatorname{Var}\left(\mu_{1}\right)+\operatorname{Var}\left(\mu_{2}\right)}$. Then,

$$
W_{2}\left(\mu_{1}, \mu_{2}\right) \leq C \sigma \sqrt{\int_{\mathbb{R}} \min \left\{\left(F^{\prime}(t)-1\right)^{2}, 1\right\} d \mu_{1}(t)}
$$

where $F$ is the monotone transportation map between $\mu_{1}$ and $\mu_{2}$ and $C>0$ is a universal constant.

We begin the proof of Proposition 5.1 with the following crude:

Lemma 5.2. Let $\mu_{1}$ and $\mu_{2}$ be probability measures on the real line.

(i) If $\mu_{1}$ and $\mu_{2}$ are even, then

$$
W_{2}\left(\mu_{1}, \mu_{2}\right)^{2} \leq 2\left(\operatorname{Var}\left(\mu_{1}\right)+\operatorname{Var}\left(\mu_{2}\right)\right)
$$

(ii) If $\mu_{1}, \mu_{2}$ are supported on $[A, \infty)$ and $[B, \infty)$ respectively, and have nonincreasing densities, then

$$
W_{2}\left(\mu_{1}, \mu_{2}\right) \leq|B-A|+10 \sqrt{\operatorname{Var}\left(\mu_{1}\right)+\operatorname{Var}\left(\mu_{2}\right)} .
$$

Proof. Denote by $\delta_{0}$ the Dirac measure at the origin. Assume that $\mu_{0}$ and $\mu_{1}$ are even. By the triangle inequality for the transportation metric,

$$
W_{2}\left(\mu_{1}, \mu_{2}\right) \leq W_{2}\left(\mu_{1}, \delta_{0}\right)+W_{2}\left(\delta_{0}, \mu_{2}\right)=\sqrt{\operatorname{Var}\left(\mu_{1}\right)}+\sqrt{\operatorname{Var}\left(\mu_{2}\right)},
$$

and (i) follows. We move on to prove (ii). Denote $e=\mathbb{E}\left[\mu_{1}\right]$. It follows from the fact that the density of $\mu_{1}$ is non-increasing that the expectation of $\mu_{1}$ is larger than its median. Hence,

$$
\mu_{1}([A, e]) \geq \frac{1}{2}, \quad \text { and } \quad \mu_{1}\left(\left[A, A+\frac{e-A}{2}\right]\right) \geq \frac{1}{4}
$$

Therefore,

$$
\operatorname{Var}\left(\mu_{1}\right) \geq \int_{A}^{A+\frac{e-A}{2}}(e-x)^{2} d \mu_{1}(x) \geq \frac{(e-A)^{2}}{16} .
$$

Let $\delta_{A}, \delta_{B}, \delta_{e}$ be the Dirac measures supported on $A, B, e$ respectively. By the triangle inequality,

$$
W_{2}\left(\mu_{1}, \delta_{A}\right) \leq W_{2}\left(\mu_{1}, \delta_{e}\right)+W_{2}\left(\delta_{e}, \delta_{A}\right)=\sqrt{\operatorname{Var}\left(\mu_{1}\right)}+(e-A) \leq 5 \sqrt{\operatorname{Var}\left(\mu_{1}\right)} .
$$

In the same manner,

$$
W_{2}\left(\mu_{2}, \delta_{B}\right) \leq 5 \sqrt{\operatorname{Var}\left(\mu_{2}\right)} .
$$

Therefore, by using $W_{2}\left(\mu_{1}, \mu_{2}\right) \leq W_{2}\left(\mu_{1}, \delta_{A}\right)+W_{2}\left(\delta_{A}, \delta_{B}\right)+W_{2}\left(\delta_{B}, \mu_{2}\right)$,

$$
W_{2}\left(\mu_{1}, \mu_{2}\right) \leq 10 \sqrt{\operatorname{Var}\left(\mu_{1}\right)+\operatorname{Var}\left(\mu_{2}\right)}+|B-A|
$$


Proof of Proposition 5.1. Use (5.1), the definition of $F$, and the fact that $\Phi_{1}^{-1}$ pushes forward the uniform measure on $[0,1]$ to $\mu_{1}$, in order to obtain

$$
\int_{\mathbb{R}} \min \left\{\left(F^{\prime}(t)-1\right)^{2}, 1\right\} d \mu_{1}(t)=\int_{0}^{1} \min \left\{\left(\frac{\rho_{1}\left(\Phi_{1}^{-1}(t)\right)}{\rho_{2}\left(\Phi_{2}^{-1}(t)\right)}-1\right)^{2}, 1\right\} d t .
$$

Recall that when $\mu_{j}$ is a log-concave measure, the function $\rho_{j}\left(\Phi_{j}^{-1}(t)\right)$ is concave on $[0,1]$. Denote $I_{j}(t)=\rho_{j}\left(\Phi_{j}^{-1}(t)\right)$ for $j=1,2$. Then $I_{1}$ and $I_{2}$ are concave, non-negative functions on $[0,1]$, with the property that $I_{j}(t)=I_{j}(1-t)$ for any $t \in[0,1]$. These two functions are therefore continuous on $(0,1)$, increasing on $[0,1 / 2]$, and decreasing on $[1 / 2,1]$. Let $\varepsilon>0$ be such that

$$
\varepsilon^{2}=\int_{0}^{1} \min \left\{\left(\frac{I_{1}(t)}{I_{2}(t)}-1\right)^{2}, 1\right\} d t
$$

Suppose first that $\varepsilon>1 / 10$. In this case, from part (i) of lemma 5.2,

$$
W_{2}\left(\mu_{1}, \mu_{2}\right)^{2} \leq 2\left(\operatorname{Var}\left(\mu_{1}\right)+\operatorname{Var}\left(\mu_{2}\right)\right) .
$$

So whenever $\varepsilon>1 / 10$, the inequality (5.3) holds trivially for a sufficiently large universal constant $C>0$.

From now on, we restrict attention to the case where $\varepsilon \leq 1 / 10$. We divide the rest of the proof into several steps.

Step 1: Let us prove that there exists a universal constant $C>0$ such that

$$
\int_{2 \varepsilon^{2}}^{1-2 \varepsilon^{2}}\left(\frac{I_{1}(t)}{I_{2}(t)}-1\right)^{2} d t \leq C \varepsilon^{2}
$$

To that end, we will show that

$$
I_{1}(t) \leq 4 I_{2}(t) \quad \text { for all } t \in\left[2 \varepsilon^{2}, 1-2 \varepsilon^{2}\right] .
$$

Once we prove (5.6), the desired bound (5.5) follows from (5.4). We thus focus on the proof of (5.6). Suppose that $t_{1} \in(0,1 / 2]$ satisfies $I_{1}\left(t_{1}\right)>4 I_{2}\left(t_{1}\right)$. We will show that in this case

$$
t_{1} \leq 2 \varepsilon^{2} \text {. }
$$

If $I_{1}(t)>2 I_{2}(t)$ for all $t \in\left(0, t_{1}\right)$, then $t_{1} \leq \varepsilon^{2}$ according to (5.4). Thus (5.7) holds true in this case. Otherwise, there exists $0<t<t_{1}$ with $I_{1}(t) \leq 2 I_{2}(t)$. Let $t_{0}$ be the supremum over all such $t$. Since $I_{1}$ and $I_{2}$ are continuous and non-decreasing on $\left(0, t_{1}\right]$, then

$$
I_{1}\left(t_{0}\right)=2 I_{2}\left(t_{0}\right) \leq 2 I_{2}\left(t_{1}\right)<I_{1}\left(t_{1}\right) / 2 .
$$

Since $I_{1}$ is concave, non-decreasing and non-negative on $\left[0, t_{1}\right]$, then necessarily $t_{0}<t_{1} / 2$. We conclude that $I_{1}(t)>2 I_{2}(t)$ for any $t \in\left[t_{1} / 2, t_{1}\right]$. From (5.4) it 
follows that $t_{1} \leq 2 \varepsilon^{2}$. Therefore (5.7) is proven in all cases. By symmetry, we conclude (5.6), and the proof of (5.5) is complete.

Step 2: For any $0 \leq T \leq \Phi_{1}^{-1}\left(1-2 \varepsilon^{2}\right)$ we have

$$
\int_{-T}^{T}\left(F^{\prime}(t)-1\right)^{2} d \mu_{1}(t) \leq \int_{2 \varepsilon^{2}}^{1-2 \varepsilon^{2}}\left(\frac{I_{1}(t)}{I_{2}(t)}-1\right)^{2} d t \leq C \varepsilon^{2},
$$

where the last inequality is the content of Step 1 . Denote $v=\left.\mu_{1}\right|_{[-T, T]}$, an even log-concave probability measure. According to Lemma 2.6, we have $\operatorname{Var}(\nu) \leq$ $\operatorname{Var}\left(\mu_{1}\right) \leq \sigma$. Note that the function $F(t)-t$ is odd, hence its $v$-average its zero. Using the Poincaré-type inequality in Lemma 2.1, we see that for any $0 \leq T \leq$ $\Phi_{1}^{-1}\left(1-2 \varepsilon^{2}\right)$,

$$
\int_{-T}^{T}(F(t)-t)^{2} d \mu_{1}(t) \leq 12 \operatorname{Var}(v) \int_{-T}^{T}\left(F^{\prime}(t)-1\right)^{2} d \mu_{1}(t) \leq \tilde{C} \sigma^{2} \varepsilon^{2} .
$$

Step 3: Let $T_{1}=\Phi_{1}^{-1}\left(1-3 \varepsilon^{2}\right)$ and let $T_{2}=\Phi_{1}^{-1}\left(1-2 \varepsilon^{2}\right)$. We use (5.8) and conclude that there exists $T_{1} \leq T \leq T_{2}$ with

$$
|F(T)-T|^{2} \leq \tilde{C} \sigma^{2} \varepsilon^{2} / \mu_{1}\left(\left[T_{1}, T_{2}\right]\right)=\tilde{C} \sigma^{2} .
$$

Denote $v_{1}=\left.\mu_{1}\right|_{[T, \infty)}$ and $v_{2}=\left.\mu_{2}\right|_{[F(T), \infty)}$. These are log-concave probability densities with $\operatorname{Var}\left(v_{1}\right)+\operatorname{Var}\left(v_{2}\right) \leq \sigma^{2}$. Note that we have, owing to (5.8),

$$
\begin{aligned}
W_{2}\left(\mu_{1}, \mu_{2}\right)^{2} & =\int_{-T}^{T}(F(t)-t)^{2} d \mu_{1}(t)+2 \int_{T}^{\infty}(F(t)-t)^{2} d \mu_{1}(t) \\
& \leq \tilde{C} \sigma^{2} \varepsilon^{2}+2 \mu_{1}([T, \infty)) W_{2}\left(v_{1}, v_{2}\right)^{2} .
\end{aligned}
$$

In order to prove the lemma it remains to show that $W_{2}\left(v_{1}, v_{2}\right)^{2} \leq C \sigma^{2}$. But in view of (5.9), the latter is a direct consequence of part (ii) in lemma 5.2: Since $T, F(T)>0$, then the log-concave densities of $v_{1}$ and $v_{2}$ are non-increasing. This completes the proof.

Let $f, g: \mathbb{R} \rightarrow[0, \infty)$ be log-concave functions with finite, positive integrals. Denote by $\mu_{f}, \mu_{g}$ the probability measures on $\mathbb{R}$ whose densities are proportional to $f$ and $g$, respectively. Let $F$ be the monotone transportation map between $\mu_{f}$ and $\mu_{g}$. Then $S(x)=(F(x)+x) / 2$ is a strictly-increasing, continuous map in $\operatorname{Supp}\left(\mu_{1}\right)$. Define

$$
h(S(x))=\sqrt{f(x) g(F(x))} \quad\left(x \in \operatorname{Supp}\left(\mu_{f}\right)\right) .
$$

We set $h(x)=0$ for any $x$ which is not in the image of $\operatorname{Supp}\left(\mu_{1}\right)$ under $S$. Then $h$ is a well-defined, non-negative, measurable function on $\mathbb{R}$. Observe that for any $x \in \mathbb{R}$,

$$
h(x) \leq \sup _{y \in \mathbb{R}} \sqrt{f(x-y) g(x+y)} .
$$


We thus view the function $h$ as a refined variant of the supremum-convolution of $f$ and $g$. The following proposition is a stability estimate for the Prékopa-Leindler inequality in one dimension. It may be viewed as the transportation-metric version of the $L^{1}$-stability estimates from Ball and Böröczky [1].

Proposition 5.3. Suppose that $f$ and $g$ are even, log-concave functions on $\mathbb{R}$ with finite, positive integrals. Denote by $\mu_{f}, \mu_{g}$ the probability measures on $\mathbb{R}$ whose densities are proportional to $f, g$ respectively. Set $\sigma=\sqrt{\operatorname{Var}\left(\mu_{f}\right)+\operatorname{Var}\left(\mu_{g}\right)}$. Then,

$$
W_{2}^{2}\left(\mu_{f}, \mu_{g}\right) \leq C \sigma^{2}\left(\frac{\int_{\mathbb{R}} h}{\sqrt{\int_{\mathbb{R}} f \int_{\mathbb{R}} g}}-1\right)
$$

where the function $h$ is defined via (5.10) and $C>0$ is a universal constant.

Proof. Multiplying the functions $f$ and $g$ by positive constants, if necessary, we may assume that $\int f=\int g=1$. Indeed, neither the left-hand side nor the righthand side of (5.3) is changed under such normalization. Let $F$ be the monotone transportation map between $\mu_{f}$ and $\mu_{g}$ and as before, $S(x)=(F(x)+x) / 2$ for $x \in \operatorname{Supp}\left(\mu_{f}\right)$. Applying the change of variables $y=S(x)$ we see that

$$
\int_{\mathbb{R}} h(y) d y=\int_{\operatorname{Supp}\left(\mu_{f}\right)} h(S(x)) S^{\prime}(x) d x=\int_{\operatorname{Supp}\left(\mu_{f}\right)} \sqrt{f(x) g(F(x))} \frac{F^{\prime}(x)+1}{2} d x .
$$

According to (5.1), we have $F^{\prime}(x) g(F(x))=f(x)$ for any $x$ in the support of $\mu_{f}$. Since $g$ is log-concave, it does not vanish in $\operatorname{Supp}\left(\mu_{g}\right)$, and hence $F^{\prime}(x) \neq 0$ for any $x \in \operatorname{Supp}\left(\mu_{f}\right)$. Therefore,

$$
\begin{aligned}
\int_{\mathbb{R}} h(y) d y & =\int_{\operatorname{Supp}\left(\mu_{f}\right)} \frac{F^{\prime}(x)+1}{2 \sqrt{F^{\prime}(x)}} f(x) d x \\
& \geq \int_{\operatorname{Supp}\left(\mu_{f}\right)}\left(1+c \min \left\{\left(F^{\prime}(x)-1\right)^{2}, 1\right\}\right) f(x) d x,
\end{aligned}
$$

where we used Lemma 3.2(ii) in the last passage. Since $\int f=1$, then

$$
\int_{\mathbb{R}} h(y) d y-1 \geq c \int_{\operatorname{Supp}\left(\mu_{f}\right)} \min \left\{\left(F^{\prime}(x)-1\right)^{2}, 1\right\} f(x) d x .
$$

We may thus apply Proposition 5.1 and deduce that

$$
\int_{\mathbb{R}} h(y) d y-1 \geq c \int_{\mathbb{R}} \min \left\{\left(F^{\prime}(x)-1\right)^{2}, 1\right\} d \mu_{f}(x) \geq \frac{\tilde{c}}{\sigma^{2}} W_{2}\left(\mu_{f}, \mu_{g}\right)^{2}
$$

and the proposition is proven. 


\section{Unconditional convex bodies}

In this section we prove Theorem 1.3 together with its close variant, Theorem 6.1 below. We say that a function $\rho$ on $\mathbb{R}^{n}$ is unconditional if it is invariant under coordinate reflections, i.e., if

$$
\rho\left(x_{1}, \ldots, x_{n}\right)=\rho\left( \pm x_{1}, \ldots, \pm x_{n}\right)
$$

for all $\left(x_{1}, \ldots, x_{n}\right) \in \mathbb{R}^{n}$ and for any choice of signs. For two functions $f, g: \mathbb{R}^{n} \rightarrow$ $[0, \infty)$ we abbreviate

$$
H(f, g)(x)=\sup _{y \in \mathbb{R}^{n}} \sqrt{f(x+y) g(x-y)} .
$$

Thus, $H(f, g)=H_{1 / 2}(f, g)$ as defined in (4.1). We will frequently consider $H(f, g)(x)$ when the functions $f$ and $g$ are defined only on a subset of $\mathbb{R}^{n}$. For the purpose of (6.1) we treat such functions as zero outside their original domain of definition.

Theorem 6.1. Let $M>0$ and consider the cube $Q^{n}=[-M, M]^{n} \subset \mathbb{R}^{n}$. Suppose that $f, g: Q^{n} \rightarrow[0, \infty)$ are unconditional, log-concave probability densities. Then,

$$
W_{2}^{2}\left(\mu_{f}, \mu_{g}\right) \leq C M^{2}\left[\int_{Q^{n}} H(f, g)-1\right],
$$

where $C>0$ is a universal constant and $\mu_{f}, \mu_{g}$ are the probability measures with densities $f$, g respectively.

The main tool in the proof of Theorem 6.1 is the Knothe map from [16], which we define next. Let $M, f, g$ be as in Theorem 6.1. Then the support of $\mu_{g}$ is a convex set, and $g$ does not vanish in $\operatorname{Supp}\left(\mu_{g}\right)$. The Knothe map between $\mu_{f}$ and $\mu_{g}$ is the continuous function $F=\left(F_{1}, \ldots, F_{n}\right): \operatorname{Supp}\left(\mu_{f}\right) \rightarrow \operatorname{Supp}\left(\mu_{g}\right)$ for which

(a) $F_{*}\left(\mu_{f}\right)=\mu_{g}$.

(b) For any $j$, the function $F_{j}\left(x_{1}, \ldots, x_{n}\right)$ actually depends only on the variables $x_{1}, \ldots, x_{j}$. We may thus speak of $F_{j}\left(x_{1}, \ldots, x_{j}\right)$.

(c) For any $j$ and for any fixed $x_{1}, \ldots, x_{j-1}$, the function $F_{j}\left(x_{1}, \ldots, x_{j}\right)$ is nondecreasing in $x_{j}$.

It may be proven by induction on $n$ (see [16]) that the Knothe map between $\mu_{f}$ and $\mu_{g}$ exists, and that in fact, the three requirements above determine the function $F$ completely. Denoting $\lambda_{j}(x)=\partial F_{j}(x) / \partial x_{j} \geq 0$, it follows from property (b) that

$$
\prod_{j=1}^{n} \lambda_{j}(x)=J_{F}(x)=\frac{f(x)}{g(F(x))}
$$


for any $x \in \operatorname{Supp}\left(\mu_{1}\right)$, where $J_{F}(x)$ is the Jacobian of the map $F$. Below we will also use the fact that the map $x \mapsto x+F(x)$, defined for $x \in \operatorname{Supp}\left(\mu_{f}\right)$, is one-to-one, as follows from properties (b) and (c). Set

$$
\pi\left(x_{1}, \ldots, x_{n}\right)=\left(x_{1}, \ldots, x_{n-1}\right)
$$

and let $f_{n-1}, g_{n-1}$ be the densities of the probability measures $\pi_{*}\left(\mu_{f}\right), \pi_{*}\left(\mu_{g}\right)$, respectively. Then $f_{n-1}$ and $g_{n-1}$ are unconditional and log-concave. Write $T_{n}=$ $F=\left(F_{1}, \ldots, F_{n}\right)$ for the Knothe map between $\mu_{f}$ and $\mu_{g}$, and set

$$
T_{n-1}\left(x_{1}, \ldots, x_{n-1}\right)=\left(F_{1}\left(x_{1}\right), F_{2}\left(x_{1}, x_{2}\right), \ldots, F_{n-1}\left(x_{1}, \ldots, x_{n-1}\right)\right) .
$$

Then $T_{n-1}$ is the Knothe map between $\pi_{*}\left(\mu_{f}\right)$ and $\pi_{*}\left(\mu_{g}\right)$. Observe that for fixed $\left(x_{1}, \ldots, x_{n-1}\right) \in \pi\left(\operatorname{Supp}\left(\mu_{f}\right)\right)$, the map

$$
x_{n} \mapsto F_{n}\left(x_{1}, \ldots, x_{n}\right)
$$

is the monotone transportation map between the probability densities proportional to

$$
t \mapsto f\left(x_{1}, \ldots, x_{n-1}, t\right) \quad \text { and } \quad s \mapsto g\left(z_{1}, \ldots, z_{n-1}, s\right),
$$

for $\left(z_{1}, \ldots, z_{n-1}\right)=T_{n-1}\left(x_{1}, \ldots, x_{n-1}\right)$. For $i=n-1, n$ we set

$$
S_{i}(x)=\frac{x+T_{i}(x)}{2}
$$

which is a one-to-one, continuous function, defined for $x \in \operatorname{Supp}\left(\mu_{f}\right)$ when $i=n$ and for $x \in \pi\left(\operatorname{Supp}\left(\mu_{f}\right)\right)$ when $i=n-1$. According to (6.3) and to property (b), the Jacobian $J_{S_{i}}(x)$ of the map $S_{i}$ satisfies

$$
J_{S_{i}}(x)=\prod_{j=1}^{i}\left(\frac{1+\lambda_{j}(x)}{2}\right) \geq \prod_{j=1}^{i} \sqrt{\lambda_{j}(x)}=\sqrt{J_{T_{i}}(x)} .
$$

Finally, for $i=n-1, n$ set

$$
V\left(f_{i}, g_{i}\right)\left(S_{i}(x)\right)=\sqrt{f_{i}(x) g_{i}\left(T_{i}(x)\right)} \leq H\left(f_{i}, g_{i}\right)\left(S_{i}(x)\right) .
$$

Since $S_{i}$ is one-to-one, then $V\left(f_{i}, g_{i}\right)$ is a well-defined function on a subset of $Q^{i}$. We extend $V\left(f_{i}, g_{i}\right)$ to the entire $Q^{i}$ by setting it to be zero outside its original domain of definition.

Lemma 6.2. Let $\varphi: Q^{n-1} \rightarrow[0, \infty)$ be a measurable function. Then,

$$
\int_{Q^{n-1}} \varphi\left(S_{n-1}(y)\right) f_{n-1}(y) d y \leq \int_{Q^{n-1}} \varphi(y) V\left(f_{n-1}, g_{n-1}\right)(y) d y .
$$


Proof. We use (6.3) for the Knothe map $T_{n-1}$ to conclude that

$$
\begin{aligned}
& \int_{Q^{n-1}} \varphi\left(S_{n-1}(y)\right) f_{n-1}(y) d y \\
& \quad=\int_{\operatorname{Supp}\left(f_{n-1}\right)} \varphi\left(S_{n-1}(y)\right) \sqrt{f_{n-1}(y) g_{n-1}\left(T_{n-1}(y)\right)} \sqrt{J_{T_{n-1}}(y)} d y \\
& \quad \leq \int_{\operatorname{Supp}\left(f_{n-1}\right)} \varphi\left(S_{n-1}(y)\right) V\left(f_{n-1}, g_{n-1}\right)\left(S_{n-1}(y)\right) J_{S_{n-1}}(y) d y
\end{aligned}
$$

where we used (6.4) and (6.5) in the last passage. The map $S_{n-1}$ is one-to-one in the support of $f_{n-1}$. Changing variables $z=S_{n-1}(y)$ we obtain

$$
\int_{Q^{n-1}} \varphi\left(S_{n-1}(y)\right) f_{n-1}(y) d y \leq \int_{S_{n-1}\left(\operatorname{Supp}\left(f_{n-1}\right)\right)} \varphi(z) V\left(f_{n-1}, g_{n-1}\right)(z) d z
$$

and the lemma is proven.

The following lemma will serve as the induction step in the proof of Theorem 6.1.

Lemma 6.3. Let $M>0, Q^{n}=[-M, M]^{n}$. Suppose that $f, g: Q^{n} \rightarrow \mathbb{R}$ are unconditional, log-concave probability densities. Let $T_{n}, T_{n-1}, f_{n-1}, g_{n-1}$ be as above. Then,

$$
\begin{aligned}
\int_{Q^{n}}\left|T_{n}(x)-x\right|^{2} f(x) d x \leq & \int_{Q^{n-1}}\left|T_{n-1}(y)-y\right|^{2} f_{n-1}(y) d y \\
& +C M^{2}\left[\int_{Q^{n}} V(f, g)-\int_{Q^{n-1}} V\left(f_{n-1}, g_{n-1}\right)\right],
\end{aligned}
$$

where $C>0$ is a universal constant (in fact, it is the same constant as in Proposition (5.3)).

Proof. In this proof we use $x=(y, t) \in \mathbb{R}^{n-1} \times \mathbb{R}$ as coordinates in $\mathbb{R}^{n}$. From the definition of $T_{n-1}$,

$$
\begin{aligned}
\int_{Q^{n}}\left|T_{n}(x)-x\right|^{2} f(x) d x= & \int_{Q^{n-1}}\left|T_{n-1}(y)-y\right|^{2} f_{n-1}(y) d y \\
& +\int_{-M}^{M} \int_{Q^{n-1}}\left|F_{n}(y, t)-t\right|^{2} f(y, t) d y d t .
\end{aligned}
$$

In order to prove the lemma, it therefore suffices to show that

$$
\begin{aligned}
& \int_{-M}^{M} \int_{Q^{n-1}}\left|F_{n}(y, t)-t\right|^{2} f(y, t) d y d t \\
& \quad \leq C M^{2}\left[\int_{Q^{n}} V(f, g)-\int_{Q^{n-1}} V\left(f_{n-1}, g_{n-1}\right)\right] .
\end{aligned}
$$


Recall that $t \mapsto F_{n}(y, t)$ is the monotone transportation map between the even, log-concave probability measures supported on $[-M, M]$, whose densities are proportional to $t \mapsto f(y, t)$ and $s \mapsto g\left(T_{n-1}(y), s\right)$. The variance of an even measure supported on $[-M, M]$ cannot exceed $M^{2}$. We may therefore use Proposition 5.3, together with (5.2), to conclude that for any $y \in \pi\left(\operatorname{Supp}\left(\mu_{f}\right)\right)$,

$$
\int_{-M}^{M}\left|F_{n}(y, t)-t\right|^{2} \frac{f(y, t)}{f_{n-1}(y)} d t \leq C M^{2}\left[\frac{\int_{-M}^{M} V(f, g)\left(S_{n-1}(y), t\right) d t}{\sqrt{f_{n-1}(y) g_{n-1}\left(T_{n-1}(y)\right)}}-1\right] .
$$

In particular, the right-hand side of (6.8) is non-negative. We use the definition (6.5) and integrate with respect to $y$. This yields:

$$
\begin{aligned}
& \int_{Q^{n-1}} \int_{-M}^{M}\left|F_{n}(y, t)-t\right|^{2} f(y, t) d t d y \\
& \quad \leq C M^{2} \int_{Q^{n-1}}\left[\frac{\int_{-M}^{M} V(f, g)\left(S_{n-1}(y), t\right) d t}{V\left(f_{n-1}, g_{n-1}\right)\left(S_{n-1}(y)\right)}-1\right] f_{n-1}(y) d y \\
& \quad \leq C M^{2} \int_{Q^{n-1}}\left[\frac{\int_{-M}^{M} V(f, g)(y, t) d t}{V\left(f_{n-1}, g_{n-1}\right)(y)}-1\right] V\left(f_{n-1}, g_{n-1}\right)(y) d y
\end{aligned}
$$

where the last passage is legal according to Lemma 6.2. The desired estimate (6.7) follows, and the proof is complete.

Proof of Theorem 6.1. We will prove by induction on the dimension $n$ that

$$
\int_{Q^{n}}\left|T_{n}(x)-x\right|^{2} f(x) d x \leq C M^{2}\left[\int_{Q^{n}} V(f, g)-1\right],
$$

where $C$ is the constant from Lemma 6.3. The case $n=1$ follows from Proposition 5.3 and from the fact that the variance of an even measure supported on $[-M, M]$ cannot exceed $M^{2}$. We assume that (6.9) is proven for dimension $n-1$ and proceed with the proof for dimension $n$. Apply the induction hypothesis for the unconditional, $\log$-concave probability densities $f_{n-1}, g_{n-1}$ and conclude that

$$
\int_{Q^{n-1}}\left|T_{n-1}(y)-y\right|^{2} f_{n-1}(y) d y \leq C M^{2}\left[\int_{Q^{n-1}} V\left(f_{n-1}, g_{n-1}\right)-1\right] .
$$

Combining (6.6) and (6.10),

$$
\begin{aligned}
& \int_{Q^{n}}\left|T_{n}(x)-x\right|^{2} f(x) d x \\
& \leq C M^{2}\left\{\left[\int_{Q^{n-1}} V\left(f_{n-1}, g_{n-1}\right)-1\right]+\left[\int_{Q^{n}} V(f, g)-\int_{Q^{n-1}} V\left(f_{n-1}, g_{n-1}\right)\right]\right\}
\end{aligned}
$$

and (6.9) is proven for dimension $n$, hence for all dimensions. Using (6.9) and the fact that $V(f, g) \leq H(f, g)$, the theorem follows by the definition of transportation distance. 
The uniform measure on a convex body is a prime example for a log-concave measure. Consequently, we may deduce Theorem 1.3 from Theorem 6.1 by using a crude "cut with a big cube" argument. The logarithmic factor of Theorem 1.3 may be an artifact of this clumsy procedure.

Proof of Theorem 1.3. Let $0 \leq \gamma \leq 1 / 2$ be a parameter to be specified later on. For $\alpha, \beta>0$ we denote

$$
K_{\alpha}=K \cap[-\alpha \log n, \alpha \log n]^{n}, \quad T_{\beta}=T \cap[-\beta \log n, \beta \log n]^{n} .
$$

According to Corollary 2.4, we have $\operatorname{Cov}\left(\mu_{T}\right) \leq C R^{4}$. Using Lemma 2.2 and a union bound, we deduce that

$$
\mu_{K}\left(K \backslash K_{\alpha}\right) \leq C n^{1-c \alpha}, \quad \mu_{T}\left(T \backslash T_{\beta}\right) \leq C n^{1-c \beta / R^{2}} .
$$

We now select $\alpha$ and $\beta$ so that

$$
\mu_{K}\left(K \backslash K_{\alpha}\right)=\mu_{T}\left(T \backslash T_{\beta}\right)=\gamma .
$$

According to (6.11),

$$
\alpha \leq C\left(1+\frac{\log (1 / \gamma)}{\log n}\right), \quad \beta \leq C R^{2}\left(1+\frac{\log (1 / \gamma)}{\log n}\right) .
$$

Denote by $\mu_{K}^{1}$ the uniform probability measure on $K_{\alpha}$ and similarly for $T$. By elementary properties of the transportation metric $W_{2}$, it follows that $W_{2}^{2}\left(\mu_{K}, \mu_{T}\right) \leq \mu_{K}\left(K_{\alpha}\right) \cdot W_{2}^{2}\left(\mu_{K}^{1}, \mu_{T}^{1}\right)+\mu_{K}\left(K \backslash K_{\alpha}\right) \cdot[\operatorname{Diam}(K)+\operatorname{Diam}(T)]^{2}$, where $\operatorname{Diam}(K)=\sup _{x, y \in K}|x-y|$ is the diameter of $K$. It is well-known (see [18]) that $\operatorname{Diam}(K) \leq C n \sqrt{\left\|\operatorname{Cov}\left(\mu_{K}\right)\right\|_{O P}}$ and therefore,

$$
W_{2}^{2}\left(\mu_{K}, \mu_{T}\right) \leq W_{2}^{2}\left(\mu_{K}^{1}, \mu_{T}^{1}\right)+C \gamma n^{2} R^{4}
$$

Note that $\mu_{K}^{1}$ and $\mu_{T}^{1}$ satisfy the requirements of Theorem 6.1 with $M=\max \{\alpha, \beta\}$. $\log n$. Denote $f(x)=1_{K_{\alpha}}(x) / \operatorname{Vol}_{n}\left(K_{\alpha}\right), g(x)=1_{T_{\beta}}(x) / \operatorname{Vol}_{n}\left(T_{\beta}\right)$. Then,

$$
\int_{\mathbb{R}^{n}} H(f, g)=\frac{\operatorname{Vol}_{n}\left(\left[K_{\alpha}+T_{\beta}\right] / 2\right)}{\sqrt{\operatorname{Vol}_{n}\left(K_{\beta}\right) \operatorname{Vol}_{n}\left(T_{\beta}\right)}} \leq \frac{R}{1-\gamma} \leq R(1+2 \gamma)=1+(R-1)+2 R \gamma .
$$

From Theorem 6.1 and (6.13) we conclude that

$$
\begin{aligned}
W_{2}^{2}\left(\mu_{K}, \mu_{T}\right) \leq & C \log ^{2} n \cdot\left[\alpha^{2}+\beta^{2}\right] \cdot\{(R-1)+2 R \gamma\}+C \gamma n^{2} R^{4} \\
\leq & C \log ^{2} n \cdot\left[R^{4}\left(1+\frac{\log (1 / \gamma)}{\log n}\right)^{2}\right] \\
& \cdot\{(R-1)+2 R \gamma\}+C \gamma n^{2} R^{4} .
\end{aligned}
$$


All that remains to do is to select $\gamma$. In the case where $R \leq n^{2}$, we choose

$$
\gamma=(R-1)^{5} \log ^{2} n /\left(10 n^{4} R^{4}\right) \leq 1 / 2
$$

and deduce the desired bound (1.10) from (6.14). In the case where $R \geq n^{2}$, we select $\gamma=1 / 2$ and still deduce (1.10). The theorem is thus proven for all cases.

Next, we explain why Theorem 1.1 provides a non-trivial estimate for the thinshell parameter, and why Theorem 1.3 provides yet another proof for the thin-shell estimate from [15], up to logarithmic factors. Observe that when $K \subset \mathbb{R}^{n}$ is a convex body and $T \subset K$, then

$$
\operatorname{Vol}_{n}\left(\frac{T+K}{2}\right) \leq \operatorname{Vol}_{n}(K)=R \sqrt{\operatorname{Vol}_{n}(K) \operatorname{Vol}_{n}(T)}
$$

for $R=\sqrt{\operatorname{Vol}_{n}(K) / \operatorname{Vol}_{n}(T)}$. As before, we write $B_{2}^{n}=\left\{x \in \mathbb{R}^{n} ;|x| \leq 1\right\}$ for the Euclidean unit ball, centered at the origin in $\mathbb{R}^{n}$.

Proposition 6.4. Let $A>0$ and let $K \subset \mathbb{R}^{n}$ be an isotropic convex body. For $s>0$ denote $K_{s}=K \cap\left(s B_{2}^{n}\right)$. Assume that

$$
\left|\frac{\int_{K_{s}}|x|^{2} d \mu_{K_{s}}(x)}{\int_{K}|x|^{2} d \mu_{K}(x)}-1\right| \leq A
$$

for any $s>0$ with $\operatorname{Vol}_{n}\left(K_{s}\right) / \operatorname{Vol}_{n}(K) \in[1 / 8,7 / 8]$. Then,

$$
\int_{K}\left(\frac{|x|^{2}}{n}-1\right)^{2} d \mu_{K}(x) \leq C A^{2}
$$

where $C>0$ is a universal constant.

Proof. Standard bounds on the distribution of polynomials on high-dimensional convex sets (see Bourgain [6] or Nazarov, Sodin and Volberg [19]) reduce the desired inequality (6.16) to the estimate

$$
\mu_{K}\left(\left\{x \in K ;\left|\frac{|x|^{2}}{n}-1\right| \geq 20 A\right\}\right) \leq \frac{1}{2}
$$

In order to prove (6.17), select $a>0$ such that $\operatorname{Vol}_{n}\left(K_{a}\right)=\operatorname{Vol}_{n}(K) / 4$. From (6.15),

$$
\max _{x \in K_{a}} \frac{|x|^{2}}{n} \geq \int_{K_{a}} \frac{|x|^{2}}{n} d \mu_{K_{a}}(x) \geq 1-A,
$$

or equivalently,

$$
\mu_{K}\left(\left\{x \in K ; \frac{|x|^{2}}{n} \leq 1-A\right\}\right) \leq \frac{1}{4}
$$


For the upper bound, let $s<t$ be such that $\operatorname{Vol}_{n}\left(K_{s}\right)=3 \operatorname{Vol}_{n}(K) / 4$ and $\operatorname{Vol}_{n}\left(K_{t}\right)=$ $7 \operatorname{Vol}_{n}(K) / 8$. Then, from (6.15),

$$
\begin{aligned}
1+A & \geq \int_{K_{t}} \frac{|x|^{2}}{n} d \mu_{K_{t}}(x) \geq \frac{6}{7} \int_{K_{s}} \frac{|x|^{2}}{n} d \mu_{K_{s}}(x)+\frac{1}{7} \max _{x \in K_{s}} \frac{|x|^{2}}{n} \\
& \geq \frac{6}{7}(1-A)+\frac{1}{7} \max _{x \in K_{s}} \frac{|x|^{2}}{n} .
\end{aligned}
$$

Hence, $\max _{x \in K_{s}} \frac{|x|^{2}}{n} \leq 1+13 A$, or equivalently,

$$
\mu_{K}\left(\left\{x \in K ; \frac{|x|^{2}}{n} \geq 1+13 A\right\}\right) \leq \frac{1}{4}
$$

It is now clear that (6.17) follows from (6.18) and (6.19).

\section{References}

[1] K. M. BALL and K. J. BÖRÖCZKY, Stability of some versions of the Prékopa-Leindler inequality, Monats. Math. 163 (2011), 1-14.

[2] S. G. BовKOV, Isoperimetric and analytic inequalities for log-concave probability measures, Ann. Probab. 27 (1999), 1903-1921.

[3] S. G. BobKov, Extremal properties of half-spaces for log-concave distributions, Ann. Probab. 24 (1996), 35-48.

[4] S. G. BoBKov, On concentration of distributions of random weighted sums, Ann. Probab. 31 (2003), 195-215.

[5] C. Borell, Complements of Lyapunov's inequality, Math. Ann. 205 (1973), 323-331.

[6] J. BourgaIn, On the distribution of polynomials on high-dimensional convex sets, In: "Geometric Aspects of Functional Analysis (1989-90)", Lecture Notes in Math., Vol. 1469, Springer, Berlin, 1991, 127-137.

[7] G. Carlier, A. Galichon and F. Santambrogio, From Knothe's transport to Brenier's map and a continuation method for optimal transport, SIAM J. Math. Anal. 41 (2009), 2554-2576.

[8] V. I. DiskANT, Stability of the solution of the Minkowski equation (in Russian), Sibirsk Mat. 14 (1973), 669-673, 696. English translation in Siberian Math. J. 14 (1973), 466-469.

[9] R. EldAN and B. KLARTAG, Pointwise estimates for marginals of convex bodies, J. Funct. Anal. 254 (2008), 2275-2293.

[10] R. EldAN and B. KLARTAG, Approximately Gaussian marginals and the hyperplane conjecture, In: Proc. of a workshop on "Concentration, Functional Inequalities and Isoperimetry", Contemporary Math., Vol. 545, Amer. Math. Soc., 2011, 55-68.

[11] A. Figalli, F. Maggi and A. Pratelli, A refined Brunn-Minkowski inequality for convex sets, Ann. Inst. H. Poincaré Anal. Non Linéaire 26 (2009), 2511-2519.

[12] A. Figalli, F. MAGGi and A. PRATELLI, A mass transportation approach to quantitative isoperimetric inequalities, Invent. Math. 182 (2010), 167-211.

[13] H. Groemer, On the Brunn-Minkowski theorem, Geom. Dedicata 27 (1988), 357-371.

[14] B. KlaRTAG, A central limit theorem for convex sets, Invent. Math. 168 (2007), 91-131.

[15] B. KLARTAG, A Berry-Esseen type inequality for convex bodies with an unconditional basis, Probab. Theory Related Fields 145 (2009), 1-33. 
[16] H. Knothe, Contributions to the theory of convex bodies, Michigan Math. J. 4 (1957), 39-52.

[17] L. LOVÁsZ and S. VEMPALA, The geometry of logconcave functions and sampling algorithms, Random Structures Algorithms 30 (2007), 307-358.

[18] V. D. MILMAN and A. PAJOR, Isotropic position and inertia ellipsoids and zonoids of the unit ball of a normed n-dimensional space, In: "Geometric Aspects of Functional Analysis" - Israel seminar, Lecture Notes in Math., Vol. 1376, Springer, 1989, 64-104.

[19] F. NaZarov, M. Sodin and A. Volberg, The geometric Kannan-Lovász-Simonovits lemma, dimension-free estimates for the distribution of the values of polynomials, and the distribution of the zeros of random analytic functions, St. Petersburg Math. J. 14 (2003), 351-366.

[20] R. OsSERMAn, Bonnesen-style isoperimetric inequalities, Amer. Math. Monthly 86 (1979), $1-29$.

[21] G. Pisier, "The Volume of Convex Bodies and Banach Space Geometry", Cambridge Tracts in Mathematics, Vol. 94, Cambridge University Press, Cambridge, 1989.

[22] C. Villani, 'Topics in Optimal Transportation", Graduate Studies in Mathematics, Vol.58, American Mathematical Society, Providence, RI, 2003.

School of Mathematical Sciences Tel-Aviv University

Tel Aviv 69978, Israel roneneld@tau.ac.il klartagb@tau.ac.il 